\title{
H $\alpha$ VARIABILITY IN PTFO 8-8695 AND THE POSSIBLE DIRECT DETECTION OF EMISSION FROM A 2 MILLION YEAR OLD EVAPORATING HOT JUPITER
}

\author{
Christopher M. Johns-Krull ${ }^{1}$, Lisa Prato ${ }^{2}$, Jacob N. McLane ${ }^{2,8,9}$, David R. Ciardi ${ }^{3,10}$, Julian C. van Eyken ${ }^{3}$, \\ Wei Chen ${ }^{1,9}$, John R. Stauffer ${ }^{4}$, Charles A. Beichman ${ }^{5,11}$, Sarah A. Frazier ${ }^{1}$, Andrew F. Boden ${ }^{6}$, \\ Maria Morales-Calderón ${ }^{7}$, AND Luisa M. Rebull ${ }^{4}$ \\ ${ }^{1}$ Department of Physics \& Astronomy, Rice University, 6100 Main St. MS-108, Houston, TX 77005, USA; cmj@ rice.edu, wc2@ rice.edu, sarah.a.frazier@ @ice.edu \\ ${ }^{2}$ Lowell Observatory, 1400 W. Mars Hill Rd., Flagstaff, AZ 86001, USA; 1prato@lowell.edu, jnmclane@astro.as.utexas.edu \\ ${ }^{3}$ NASA Exoplanet Science Institute (NEXScI), Caltech M/S 100-22, Pasadena, CA 91125, USA; ciardi@ipac.caltech.edu, vaneyken@ipac.caltech.edu \\ ${ }^{4}$ Spitzer Science Center/Caltech, 1200 East California Boulevard, Pasadena, CA 91125, USA; stauffer@ipac.caltech.edu, rebull@ipac.caltech.edu \\ 5 Jet Propulsion Laboratory, California Institute of Technology, 4800 Oak Grove Drive, Pasadena, CA 91109, USA; chas@ipac.caltech.edu \\ ${ }^{6}$ Division of Physics, Math and Astronomy, California Institute of Technology, 1200 E California Blvd., Pasadena, CA 91125, USA; bode@astro.caltech.edu \\ ${ }^{7}$ Centro de Astrobiología, INTA-CSIC, ESAC Campus, P.O. Box 78, E-28691 Villanueva de la Canada, Spain; mariamc@cab.inta-csic.es \\ Received 2015 September 14; revised 2016 May 11; accepted 2016 May 19; published 2016 October 3
}

\begin{abstract}
We use high time cadence, high spectral resolution optical observations to detect excess $\mathrm{H} \alpha$ emission from the 2-3 Myr old weak-lined T Tauri star PTFO 8-8695. This excess emission appears to move in velocity as expected if it were produced by the suspected planetary companion to this young star. The excess emission is not always present, but when it is, the predicted velocity motion is often observed. We have considered the possibility that the observed excess emission is produced by stellar activity (flares), accretion from a disk, or a planetary companion; we find the planetary companion to be the most likely explanation. If this is the case, the strength of the $\mathrm{H} \alpha$ line indicates that the emission comes from an extended volume around the planet, likely fed by mass loss from the planet which is expected to be overflowing its Roche lobe.
\end{abstract}

Key words: accretion, accretion disks - line: profiles - stars: atmospheres - stars: formation - stars: magnetic field - stars: pre-main sequence

\section{INTRODUCTION}

The number of known and candidate extrasolar planets continues to grow. As of 2016 May 11 there are $~ 3200$ confirmed planets and $\sim 2400$ planet candidates. ${ }^{12}$ The vast majority of these known and candidate planets have been discovered around middle-aged main sequence stars, and many of these discoveries have challenged our understanding of planet formation, starting with the discovery of the first extrasolar planet orbiting a Sun-like star (51 Peg b; Mayor \& Queloz 1995), the first of the class of "hot Jupiters." The wide variety of extrasolar planetary systems now known has led to increased interest and debate over the processes that lead to planet formation. The core nucleated accretion model (e.g., Pollack et al. 1996; Bodenheimer et al. 2000; Hubickyj et al. 2005; Lissauer \& Stevenson 2007, p. 591) produces Jupiter mass objects slowly as they are built up as a result of collisions of dust and ice particles which stick together and gradually form larger and larger bodies until sufficient mass is obtained in order to gravitationally accrete large amounts of gas from the disk. The competing gravitational instability model (e.g., Kuiper 1951; Cameron 1978; Boss 1997, 1998; Durisen

\footnotetext{
${ }^{8}$ Present address: Department of Physics \& Astronomy, Northern Arizona University, Flagstaff, AZ 86011, USA.

${ }^{9}$ Now at Department of Astronomy, The University of Texas at Austin, Austin, TX 78712, USA.

${ }^{10}$ Visiting Astronomer, Kitt Peak National Observatory, National Optical Astronomy Observatory, which is operated by the Association of Universities for Research in Astronomy (AURA) under cooperative agreement with the National Science Foundation.

11 NASA Exoplanet Science Institute (NExScI), California Institute of Technology, 770 S. Wilson Ave, Pasadena, CA 91125, USA.

${ }^{12}$ See http://exoplanets.org; http://planetquest.jpl.nasa.gov; http:// exoplanetarchive.ipac.caltech.edu/.
}

et al. 2007, p. 607) posits circumstellar disks which are massive enough to fragment as a result of their own gravity and form Jupiter mass planets on a much more rapid timescale than is typical in conventional core accretion models.

Both models of planet formation find support and difficulties with current observations. For example, the planet metallicity correlation (Gonzalez 1997; Santos et al. 2004; Fischer \& Valenti 2005) is often quoted as evidence in support of the core accretion model. On the other hand, the direct imaging discovery of massive planets on wide orbits (e.g., HR 8799b, c, \& d, Marois et al. 2008) has been taken as evidence that gravitational instabilities must be important for forming at least some planets that cannot be easily explained by the core accretion scenario (Dodson-Robinson et al. 2009). A chief limitation for the core accretion model is the timescale involved relative to the lifetime of circumstellar accretion disks. Neither the core accretion model nor the gravitational instability model originally predicted the existence of hot Jupiters, leading to the need for a mechanism such as migration to move massive planets from their distant formation sites to final positions close to the star (e.g., Levison et al. 2007, p. 669; Papaloizou et al. 2007 , p. 655). As a result, there is a significant need to establish the timescale of planet formation and planet migration. The candidate young hot Jupiter studied here could potentially set important constraints on our understanding of these processes.

A desirable way to study the planet formation process, its timescale, and the role of migration and other phenomena is to search for planets around young stars that are in the process of forming their planetary systems. Detecting this youngest generation ( few Myr old) of planets presents special challenges. The youngest stars, many still surrounded by the circumstellar material from which planets are presumed to be actively forming, are mostly located in regions at distances of 
$>100 \mathrm{pc}$. Thus, these targets are inherently faint and are further obscured and reddened by material local to the star-forming region. Young pre-main sequence stars have very strong magnetic fields (e.g., Johns-Krull 2007) and possess large starspots (e.g., Hatzes 1995). This makes detection of extrasolar planets through radial velocity (RV) monitoring difficult because starspots can introduce periodic RV signals that mimic those produced by planetary companions (e.g., Saar \& Donahue 1997). Nevertheless, several RV searches for planetary mass companions have been or are currently being conducted around low-mass, relatively young stars, including pre-main sequence stars (Esposito et al. 2006, p. 127; Paulson \& Yelda 2006; Huerta et al. 2007; Setiawan et al. 2007, 2008; Crockett et al. 2011, 2012; Nguyen et al. 2012). To date, these studies have yielded one planet around the $100 \mathrm{Myr}$ old G1-G1.5 V star HD 70573 (Setiawan et al. 2007). A planet has also been claimed around the $10 \mathrm{Myr}$ old classical T Tauri star (CTTSs) TW Hya; however, additional study of this object suggests the RV signal from the putative planet is actually caused by spot-induced RV jitter (Huélamo et al. 2008). Significant spot-induced periodic RV variability has been detected in a few additional young stars (Prato et al. 2008; Mahmud et al. 2011), highlighting the challenges of this technique when applied to young stars.

Potential planetary mass objects have recently been found around young stars through direct imaging studies (Neuhäuser et al. 2005; Luhman et al. 2006; Lafrenière et al. 2008; Schmidt et al. 2008; Ireland et al. 2011; Kraus \& Ireland 2012; Bowler et al. 2013; Delorme et al. 2013; Kraus et al. 2014). These objects are typically at orbital separations of $\geqslant 50$ au with estimated masses of several $M_{\text {JUP. }}$. These objects also challenge our models of planet formation, particularly the core accretion model, as the timescale to form planets at such large distances in a disk is expected to be about an order of magnitude greater than the estimated age of these objects (Pollack et al. 1996). The mass estimates for these objects come from comparing their estimated luminosity and temperature with theoretical evolutionary models. The theoretical models are uncertain at these young ages, and the observations required to pin down the luminosity and temperature have a number of challenges, resulting in considerable uncertainty in the final mass estimate for a given object. As an example, the companion to GQ Lup discovered by Neuhäuser et al. (2005) has mass estimates that range from $1 M_{\mathrm{JUP}}$ on the low side to $\sim 40 M_{\mathrm{JUP}}$ on the high side (e.g., Neuhäuser et al. 2008). As we attempt to advance our observational and theoretical understanding of planet and brown dwarf formation, it will be important to obtain strong limits on the mass of potential companions to young stars. Such strong mass constraints are the forte of RV measurements of extrasolar planets, particularly for those with independent constraints on the orbital inclination.

Transiting extrasolar planets offer several advantages for the study of sub-stellar mass companions to stars (e.g., Charbonneau et al. 2007, p. 701). Of primary advantage is that the inclination is well characterized, allowing for a more certain mass determination. Additionally, the radius and hence density of the planet can be determined, and numerous additional follow-up observations are possible, at least in principle. Several transit searches for extrasolar planets around young stars have now been performed (Aigrain et al. 2007; Miller et al. 2008; Neuhäuser et al. 2011; van Eyken et al. 2011; Cody et al. 2013, 2014). Very recently, a candidate transiting extrsolar planet candidate has been reported around a lowmass young ( 3 Myr) star (PTFO 8-8695; also known as CVSO 30, see Briceno et al. 2005) in the Orion OB1a/25-Ori region (van Eyken et al. 2012). This discovery paper suggests a planet with a mass $\leqslant 5.5 \pm 1.4 M_{\mathrm{JUP}}$ and a radius of $1.91 \pm 0.21 R_{\mathrm{JUP}}$ in a 0.45 day orbit around a $0.34-0.44 M_{\odot}$ M3 (Briceño et al. 2005) weak-lined (non accreting) T Tauri star (WTTS). The discovery observations noted unusual changes in the transit light curve from one observing season to the next, which Barnes et al. (2013) argue could be the result of mutual precession of the stellar rotation axis and the planet's orbital axis resulting from tidal interaction of the planet with an oblate star. The analysis of Barnes et al. suggests a likely planet mass of 3.0 or $3.6 M_{\mathrm{JUP}}$ and radius of 1.64 or $1.68 R_{\mathrm{JUP}}$ depending on the assumed mass of the star. Follow-up transit and stellar RV observations by Ciardi et al. (2015) lend support to this hypothesis.

Here, we report on high spectral resolution optical observations of PTFO 8-8695 densely sampled over a few orbital periods. We clearly detect excess $\mathrm{H} \alpha$ emission that moves in $\mathrm{RV}$ as predicted by the expected orbit of the companion, providing further evidence for the existence the planet. The $\mathrm{H} \alpha$ luminosity associated with the planet is almost equal to that coming from the star, indicating that the $\mathrm{H} \alpha$ emission volume is substantially larger than the planet itself. The most likely explanation is that the planet is losing mass at a subtantial rate, although at this time we are not able to fully rule out a small amount of accretion-related emission from a very low-mass disk that may remain around this young star. In Section 2 we describe the observations of this system, in Section 3 present our analysis of the data, and in Section 4 provide a discussion of these results, which are summarized in Section 5.

\section{OBSERVATIONS}

\subsection{Hobby-Eberly Telescope and Keck Spectroscopy}

Included in the discovery paper of van Eyken et al. (2012) is a set of high-resolution echelle spectra of PTFO 8-8695 taken at the HET (Ramsey et al. 1998) and at the Keck I telescope. At the HET the High Resolution Spectrograph (HRS Tull 1998) was employed, while the High Resolution Echelle Spectrometer (Vogt et al. 1994) was used at Keck. The details of the observations and data reduction procedures can be found in van Eyken et al. (2012). The spectral resolution of these observations is $\sim 15,000$ at the HET and $\sim 60,000$ at Keck. These data were used by van Eyken et al. (2012) to study the RV variability of PTFO 8-8695. Here, we use these observations to investigate the variability of the $\mathrm{H} \alpha$ emission line.

\subsection{McDonald Observatory}

Observations of PTFO 8-8695 were taken at the McDonald Obervatory $2.7 \mathrm{~m}$ Harlan J. Smith telescope with the Robert G. Tull Coudé echelle spectrograph (Tull et al. 1995) on UT 2013 November 15. A 1!"2 slit was used with the E2 grating to give a spectral resolution of $R \sim 61,400$ (with $\sim 2.05$ pixels per resolution element) for all observations. Approximately 50 orders with $\sim 100 \AA$ per order were dispersed across the $2080 \times 2048$ Tektronix CCD, covering the wavelength region 3400-10900 $\AA$. Integration times for all PTFO 8-8695 observations at McDonald Observatory were $2400 \mathrm{~s}$, and the seeing was $\sim 2^{\prime \prime}$ on average. Because of the faintness of the target ( $V=16.26$, van Eyken et al. 2012), the signal-to-noise 
Table 1

McDonald Observing Log for 2013 November 15

\begin{tabular}{|c|c|c|c|c|c|c|}
\hline $\begin{array}{l}\text { UT } \\
\text { Time }^{a}\end{array}$ & $\mathrm{~S} / \mathrm{N}^{\mathrm{b}}$ & $\begin{array}{c}\text { Excess } \mathrm{H} \alpha \\
W_{\text {eq }}(\AA)\end{array}$ & $\begin{array}{c}\text { Excess } \mathrm{H} \alpha \\
\sigma_{D}\left(\mathrm{~km} \mathrm{~s}^{-1}\right)\end{array}$ & $\begin{array}{c}\text { Excess } \mathrm{H} \alpha \\
\mathrm{RV}\left(\mathrm{km} \mathrm{s}^{-1}\right)\end{array}$ & Phase $^{c}$ & $\begin{array}{l}\text { Pred. Planet } \\
\text { RV }\left(\mathrm{km} \mathrm{s}^{-1}\right)^{\mathrm{c}}\end{array}$ \\
\hline $05: 43$ & 3.2 & $8.8 \pm 0.3$ & 86.5 & $189.6 \pm 9.4$ & 0.282 & 195.1 \\
\hline 08:07 & 4.3 & $9.2 \pm 0.2$ & 120.3 & $34.4 \pm 4.0$ & 0.505 & -6.2 \\
\hline $08: 53$ & 4.7 & $7.3 \pm 0.2$ & 104.4 & $-58.8 \pm 4.2$ & 0.577 & -98.2 \\
\hline 09:44 & 5.7 & $5.6 \pm 0.2$ & 59.3 & $-156.5 \pm 6.1$ & 0.656 & -164.8 \\
\hline $10: 32$ & 5.1 & $8.2 \pm 0.2$ & 84.8 & $-175.4 \pm 5.9$ & 0.730 & -197.1 \\
\hline $11: 17$ & 4.4 & $8.2 \pm 0.2$ & 80.9 & $-190.6 \pm 7.7$ & 0.800 & -189.0 \\
\hline
\end{tabular}

Notes.

${ }^{\text {a }}$ UT time at the midpoint of the exposure.

${ }^{b}$ Signal-to-noise per pixel in the continuum near $\mathrm{H} \alpha$.

${ }^{c}$ Based on ephemeris in van Eyken et al. (2012).

ratio obtained is quite low. Nevertheless, significant information can be extracted from the $\mathrm{H} \alpha$ emission line of this star. Table 1 gives a full $\log$ of the PTFO 8-8695 observations obtained on 2013 November 15.

We also use a spectrum of the dM3e flare star AD Leo as an example of the $\mathrm{H} \alpha$ profile shape of a chromospherically active M star of the same spectral type with strong emission lines. This spectrum was obtained with the same telescope and instrument, but on UT 1995 November 8. For this observation, the CCD was placed at the F1 focus (as opposed to the F3 focus for PTFO 8-8695). A 0". 59 slit was used to observe AD Leo, yielding a spectral resolution $R \sim 120,000$ spread across $\sim 4$ pixels. The same CCD was used, resulting in only 19 partial ( $\sim 23 \AA$ ) orders, including the one containing the $\mathrm{H} \alpha$ line, being recorded.

\subsection{Kitt Peak Observatory}

Observations of PTFO 8-8695 were also taken at the Kitt Peak National Obervatory $4 \mathrm{~m}$ Mayall telescope with the echelle spectrograph on the nights of UT 2012 December 8-10. A $1 . " 5$ slit was used with the $58.5-63^{\circ}$ grating to give a spectral resolution of $R \sim 25,500$ (with $\sim 3.24$ pixels per resoultion element) for all observations. The slit length projected to 9 !! 73 on the sky. Approximately 21 orders with $\sim 150 \AA$ per order were dispersed across the $2080 \times 2048$ Tektronix CCD which was binned by a factor of 2 in the cross-dispersion direction resulting in $2080 \times 1024$ images. The observed spectra covered the wavelength range $\sim 5500-8600 \AA$. Integration times for the Mayall observations of PTFO 8-8695 observations ranged from 600 to $1200 \mathrm{~s}$ and were typically taken in groups of three exposures with a Thorium-Argon lamp exposures taken at the begining of each group. The seeing varied during the run but was typically $\sim 2^{\prime \prime}$. Again, the signalto-noise ratio obtained is relatively low. A full log of the PTFO 8-8695 observations made at Kitt Peak is given in Table 2.

\subsection{Data Reduction}

All spectra were reduced with custom IDL echelle reduction routines that have been broadly described by Valenti (1994) and Hinkle et al. (2000). The reduction procedure is quite standard and includes bias subtraction, flat fielding by a normalized flat spectrum, scattered light subtraction, and optimal extraction of the spectrum. The blaze function of the echelle spectrometer is removed to first order by dividing the extracted stellar spectra by an extracted spectrum of the flat lamp. Final continuum normalization was accomplished by fitting a low-order polynomial to the blaze corrected spectra in the regions around the lines of interest for this study. For the Mayall spectra, there was room on the CCD where sky spectra are recorded above and below the stellar spectrum. A sky spectrum was extracted $\sim 3^{\prime \prime}$ above or below (depending on how well centered the star was) the stellar spectrum. The resulting sky spectrum was scaled to match the sky lines away from $\mathrm{H} \alpha$ and was then subtracted from the object spectrum. As shown below, the features of interest for this study are much broader than sky lines, so failure to subtract sky from the McDonald observations should not have any significant impact on the final results. The wavelength solution for the McDonald data was determined by fitting a two-dimensional polynomial to $n \lambda$ as function of pixel and order number, $n$, for approximately 1800 (for the F3 focus) or 100 (for the F1 focus) extracted thorium lines observed from an internal lamp assembly. The wavelength solution for the Kitt Peak data was determined for the $\mathrm{H} \alpha$ order only and utilized a third order polynomial fit to 13 extracted Thorium lines in this order.

\section{ANALYSIS}

\subsection{HET and Keck Data}

Figure 1 shows the four $\mathrm{H} \alpha$ profiles collected at the HET and the five $\mathrm{H} \alpha$ profiles collected at Keck. Each profile is labelled by the relative phase of the suspected planet (phase of zero is mid-transit), and time runs down in the figure. These profiles were collected over two months in early 2011 . Only one profile was collected on any given night, so at least two orbital cycles occur between any two of the observed profiles. To aid in keeping track of the time elapsed between these observations, the phases include non-zero values for the integer part of the phase which represents how may orbits have occured since the first of these exposures. Many of the profiles in Figure 1 show an essentially symmetric emission profile about line center with a narrow core on top of a broader emission base, similar to that seen in rapidly rotating, chromospherically active dMe stars (e.g., Jones et al. 1996). Other profiles show significantly redor blueshifted emission in addition to this centered apparent chromospheric emission. There is not an obvious relationship between the location of this excess emission and the predicted velocity position (shown as the red vertical line) of the candidate planetary companion. Overall, Figure 1 shows that there is substantial $\mathrm{H} \alpha$ line profile variability; however, given the generally large time delay in orbital cycles from one observation to the next, it is difficult to understand the source of 
Table 2

Kitt Peak Observing Log

\begin{tabular}{|c|c|c|c|c|c|c|c|c|}
\hline $\begin{array}{l}\text { UT } \\
\text { Time }^{a}\end{array}$ & $N_{\text {exp }}$ & $\begin{array}{l}\text { Ind. Exp. } \\
\text { Time (s) }\end{array}$ & $\mathrm{S} / \mathrm{N}^{\mathrm{b}}$ & $\begin{array}{c}\text { Excess } \mathrm{H} \alpha \\
W_{\text {eq }}(\AA)\end{array}$ & $\begin{array}{c}\text { Excess } \mathrm{H} \alpha \\
\sigma_{D}\left(\mathrm{~km} \mathrm{~s}^{-1}\right)\end{array}$ & $\begin{array}{c}\text { Excess } \mathrm{H} \alpha \\
\mathrm{RV}\left(\mathrm{km} \mathrm{s}^{-1}\right)\end{array}$ & Phase $^{c}$ & $\begin{array}{l}\text { Pred. Planet } \\
\mathrm{RV}\left(\mathrm{km} \mathrm{s}^{-1}\right)^{\mathrm{c}}\end{array}$ \\
\hline & & & & & 2012 Dec 8 & & & \\
\hline $04: 33$ & 3 & 600 & 7.2 & $\ldots$ & $\ldots$ & $\ldots$ & 0.484 & 20.0 \\
\hline $05: 16$ & 3 & 600 & 6.9 & $\ldots$ & $\ldots$ & $\ldots$ & 0.551 & -62.7 \\
\hline 06:02 & 3 & 600 & 6.8 & $\ldots$ & $\ldots$ & $\ldots$ & 0.621 & -137.2 \\
\hline $06: 46$ & 3 & 600 & 7.7 & $\ldots$ & $\ldots$ & $\ldots$ & 0.689 & -184.7 \\
\hline 07:29 & 3 & 600 & 7.8 & $\ldots$ & $\ldots$ & $\ldots$ & 0.756 & -199.0 \\
\hline $08: 13$ & 3 & 600 & 5.8 & $\ldots$ & $\ldots$ & $\ldots$ & 0.824 & -178.0 \\
\hline 09:14 & 3 & 600 & 8.7 & $\ldots$ & $\ldots$ & $\ldots$ & 0.919 & -97.0 \\
\hline 09:57 & 3 & 600 & 7.4 & $\ldots$ & $\ldots$ & $\ldots$ & 0.986 & -17.5 \\
\hline $10: 39$ & 3 & 600 & 7.9 & $\ldots$ & $\ldots$ & $\ldots$ & 0.051 & 62.7 \\
\hline $11: 21$ & 3 & 600 & 7.2 & $\ldots$ & $\ldots$ & $\ldots$ & 0.115 & 131.7 \\
\hline \multirow[t]{2}{*}{$11: 55$} & 2 & 600 & 6.1 & $\ldots$ & $\ldots$ & $\ldots$ & 0.169 & 173.9 \\
\hline & & & & & 2012 Dec 9 & & & \\
\hline $04: 35$ & 3 & 600 & 5.5 & $\ldots$ & $\ldots$ & $\ldots$ & 0.719 & -195.3 \\
\hline $05: 23$ & 3 & 600 & 7.4 & $\ldots$ & $\ldots$ & $\ldots$ & 0.792 & -192.2 \\
\hline $06: 11$ & 3 & 600 & 6.2 & $\ldots$ & $\ldots$ & $\ldots$ & 0.867 & -147.7 \\
\hline $06: 55$ & 3 & 600 & 2.6 & $\ldots$ & $\ldots$ & $\ldots$ & 0.935 & -79.1 \\
\hline $07: 42$ & 3 & 600 & 3.1 & $\ldots$ & $\ldots$ & $\ldots$ & 0.008 & 10.0 \\
\hline $08: 21$ & 2 & 600 & 6.4 & $\ldots$ & $\ldots$ & $\ldots$ & 0.068 & 82.5 \\
\hline 09:00 & 3 & 600 & 4.1 & $\ldots$ & $\ldots$ & $\ldots$ & 0.128 & 143.4 \\
\hline 09:47 & 3 & 600 & 6.8 & $\ldots$ & $\ldots$ & $\ldots$ & 0.201 & 189.7 \\
\hline $10: 38$ & 3 & 600 & 5.5 & $\ldots$ & $\ldots$ & $\ldots$ & 0.280 & 195.6 \\
\hline \multirow[t]{2}{*}{$11: 27$} & 3 & 600 & 6.8 & $\ldots$ & $\ldots$ & $\ldots$ & 0.356 & 156.6 \\
\hline & & & & & 2012 Dec 10 & & & \\
\hline $04: 25$ & 1 & 1200 & 4.4 & $0.3 \pm 0.2$ & $\ldots$ & $\ldots$ & 0.902 & -115.0 \\
\hline $04: 48$ & 1 & 1200 & 5.2 & $-0.1 \pm 0.2$ & $\ldots$ & $\ldots$ & 0.931 & -83.6 \\
\hline $05: 27$ & 1 & 1200 & 7.1 & $1.8 \pm 0.2$ & 83.4 & $57.3 \pm 50.0$ & 0.968 & -39.8 \\
\hline $05: 53$ & 1 & 1200 & 7.3 & $3.6 \pm 0.2$ & 92.3 & $99.4 \pm 15.2$ & 0.026 & 32.4 \\
\hline $06: 15$ & 1 & 1200 & 8.5 & $4.7 \pm 0.2$ & 111.4 & $174.0 \pm 11.5$ & 0.068 & 82.5 \\
\hline $06: 45$ & 1 & 1200 & 7.2 & $6.4 \pm 0.2$ & 101.6 & $272.4 \pm 10.9$ & 0.102 & 160.3 \\
\hline $07: 12$ & 1 & 1200 & 8.1 & $5.7 \pm 0.2$ & 97.2 & $265.9 \pm 10.0$ & 0.149 & 185.1 \\
\hline $07: 34$ & 1 & 1200 & 7.0 & $4.7 \pm 0.2$ & 88.6 & $251.7 \pm 12.6$ & 0.225 & 196.6 \\
\hline 08:03 & 1 & 1200 & 6.0 & $3.8 \pm 0.2$ & 86.4 & $245.9 \pm 17.2$ & 0.269 & 197.7 \\
\hline $08: 25$ & 1 & 1200 & 7.1 & $2.7 \pm 0.2$ & 62.3 & $202.4 \pm 16.8$ & 0.303 & 188.2 \\
\hline 08:47 & 1 & 1200 & 7.6 & $2.6 \pm 0.2$ & 69.1 & $198.8 \pm 16.3$ & 0.337 & 170.1 \\
\hline
\end{tabular}

Notes.

${ }^{\text {a }}$ UT time at the midpoint of exposure(s).

b Signal-to-noise per pixel in the continuum near $\mathrm{H} \alpha$.

${ }^{c}$ Based on ephemeris in van Eyken et al. (2012).

this variability without the inclusion of data sets with more dense temporal sampling.

\subsection{McDonald Data}

Figure 2 shows all the observed profiles of PTFO 8-8695 obtained on 2013 November 15 at McDonald Observatory. Each profile is identified with the UT time of the midpoint of the exposure. Also shown is the velocity position expected for the planetary companion based on the ephemeris published in van Eyken et al. (2012). There is clear $\mathrm{H} \alpha$ emission in the profile that appears to be moving in velocity space with the expected position of the planet. In addition, there is strong, centrally peaked $\mathrm{H} \alpha$ emission. PTFO 8-8695 is a WTTS (Briceño et al. 2005; Hernández et al. 2007) and as such its $\mathrm{H} \alpha$ emission is expected to be chromospheric in origin, that is, it is believed to be produced by the magnetic activity of the star itself (Bertout 1989). In order to estimate the stellar contribution to the observed line profiles, we used two different observed profiles from Figure 2. In the profile taken at UT 5:43, the planetary emission appears to be confined to the red side of the line profile. The profile taken at UT 10:32 is the one in which the planet is expected to be the most blueshifted, and the excess emission appears to be confined to the blue side of the line profile. The red side of the line profile in both the UT 10:32 and UT 11:17 are nearly identical, further indicating that the potential planetary emission is confined to the blue side of the profile. Therefore, to estimate the stellar chromospheric component of the line, we take the blue side of the UT 5:43 profile and combine it with the red side of the UT 10:32 profile to get the final profile shown in Figure 3. We measured the $\mathrm{H} \alpha$ equivalent width of this stellar emission, finding a value of $10.49 \pm 0.21 \AA$. Also shown in Figure 3 is the line profile of AD Leo, a dM3e flare star rotationally broadened to the same $v \sin i \quad\left(80.6 \mathrm{~km} \mathrm{~s}^{-1}\right.$ van Eyken et al. 2012) observed in PTFO 8-8695. Barnes et al. (2013) predict that the apparent $v \sin i$ of PTFO 8-8695 will change by $\sim 13 \%$ as the result of 


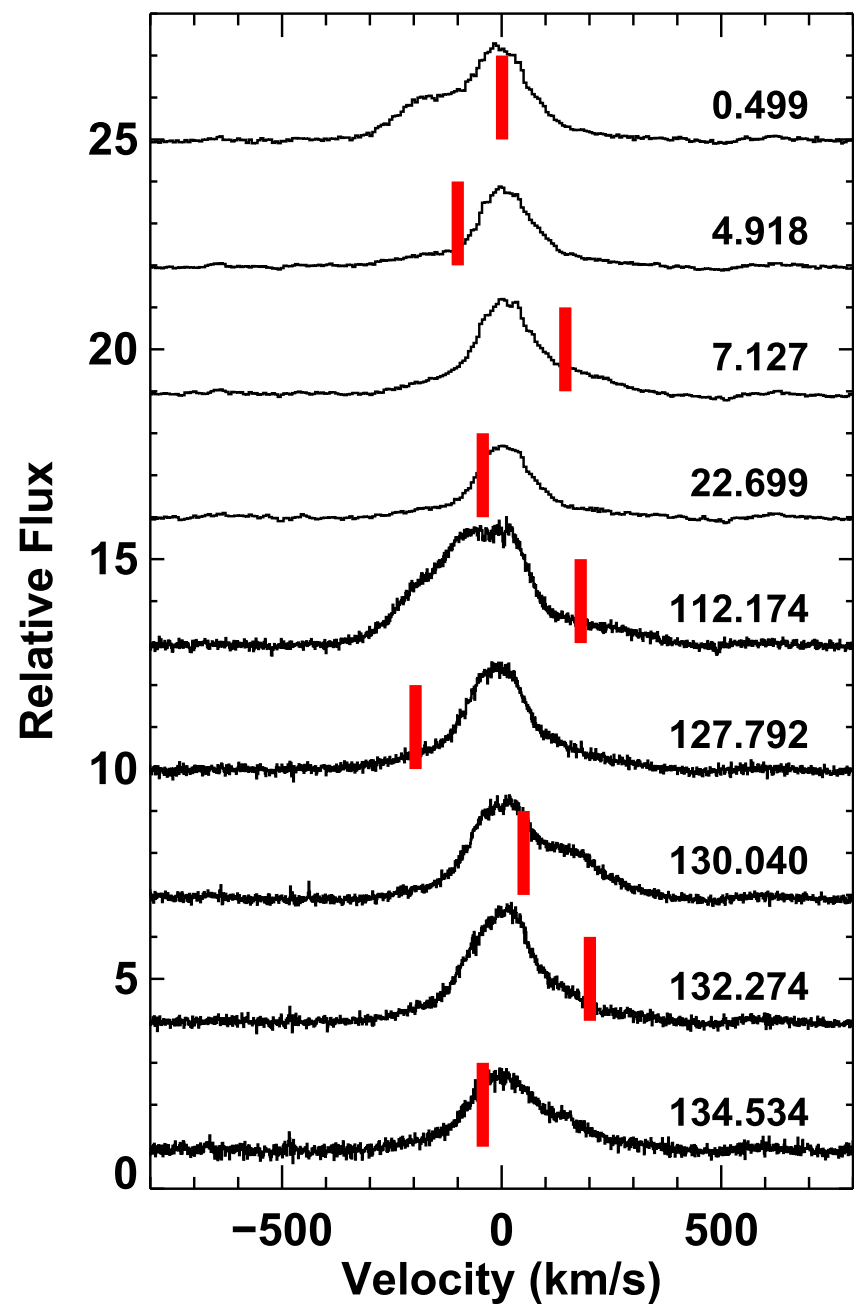

Figure 1. Continuum-normalized $\mathrm{H} \alpha$ profiles of PTFO 8-8695 obtained with the HET (top four profiles) and with Keck (bottom five profiles), as part of the discovery study of van Eyken et al. (2012). The orbital phase (with 0.0 the midpoint of the first transit to precede the first HET observation) at the midpoint of the observation is given with each profile. The thick, red vertical line on each profile marks the expected velocity position of the planetary companion detected by van Eyken et al. (2012) from transit observations of this WTTS. There is clear variability in the $\mathrm{H} \alpha$ emission.

precession of the stellar rotation axis. This effect was searched for by Yu et al. (2015) and was not seen, although they only had two observing epochs. The exact value we use for $v \sin i$ may be slightly off; however, we see no evidence that this is the case. The width of the rotationally broadened AD Leo spectrum is similar to the reconstructed "chromospheric" profile of PTFO 8-8695, but is weaker. Multiplying the AD Leo emission component by a factor of 2.4 leads to the smooth solid profile in Figure 3, which provides a reasonably good match to the PTFO 8-8695 profile, suggesting that the profile presented in Figure 3 is a good representation of the stellar component of the line profile.

Figure 4 shows each of the observed profiles from Figure 2 with the stellar profile from Figure 3 (the histogram) subtracted off. The leftover emission from this subtraction process could be entirely due to the planetary companion. Examining Figure 4 shows that the centroid of this emission tracks very well the predicted velocity position of the planet. However, there is a slight hint that the $\mathrm{H} \alpha$ emission is not quite at the predicted velocity from the ephemeris. In order to characterize this

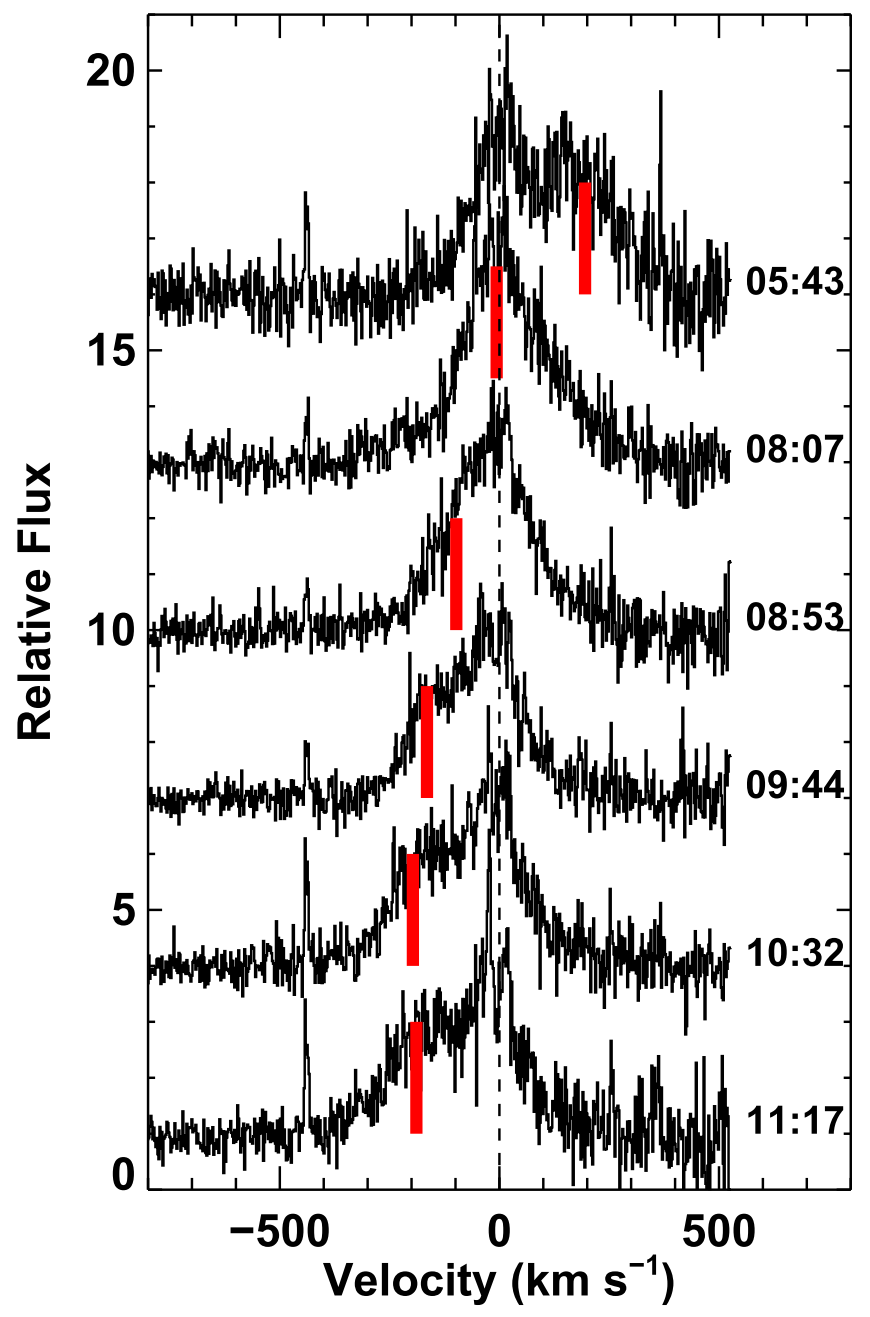

Figure 2. Six observed continuum-normalized $\mathrm{H} \alpha$ profiles of PTFO 8-8695 obtained on 2013 November 15 UT at McDonald Observatory are shown with the UT time of the midpoint of each observation given. The thick, red vertical line on each profile marks the expected velocity position of the planetary companion detected by van Eyken et al. (2012) from transit observations of this WTTS. There is clear excess $\mathrm{H} \alpha$ emission coincident in velocity space at the radial velocity of the planet.

apparent planetary emission, we measure the $\mathrm{H} \alpha$ equivalent width of each profile in Figure 4, as well as the velocity centroid of the emission and the velocity width of each profile. All these values are reported in Table 1 . For the velocity centroid we compute the flux (above the continuum) weighted mean velocity from the data. For the line width, $\sigma_{D}$, we use a measure of dispersion given by

$$
\sigma_{D}=\left(\frac{\Sigma\left(v-v_{o}\right)^{2}\left(F_{\lambda}-1\right)}{\Sigma\left(F_{\lambda}-1\right)}\right)^{1 / 2}
$$

where $v$ is the velocity of each channel in the continuumnormalized spectrum difference spectrum $F_{\lambda}$ from Figure 4, and $v_{o}$ is the previously determined velocity centroid. This measure of line width has the advantage of being purely empirical and does not rely on fitting any particular functional form to the data. In the case of a Gaussian profile, this $\sigma_{D}$ is equal to $\sigma$ in the standard Gaussian formula. There is some indication that the dispersions of the measured profiles are somewhat larger for the times when the planetary velocity 


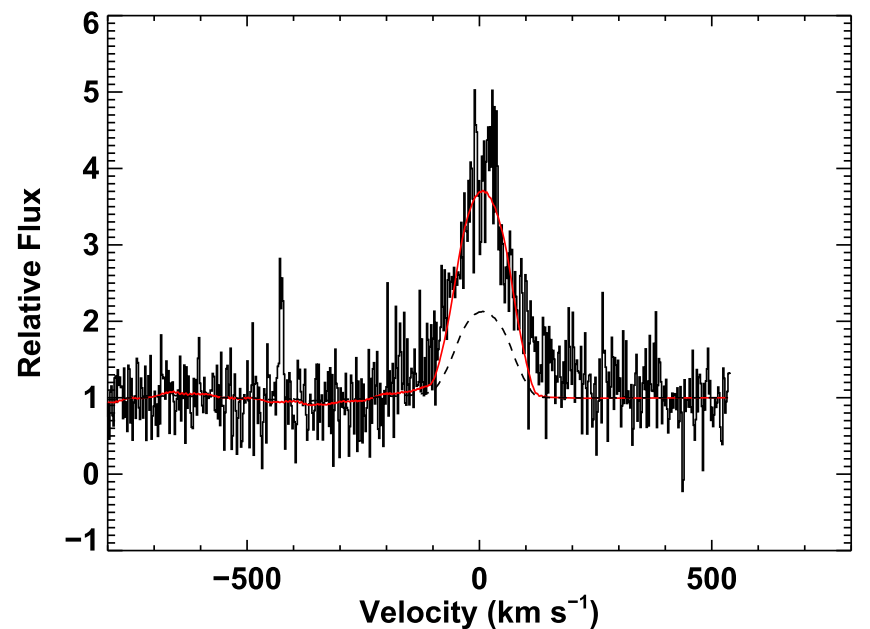

Figure 3. Black histogram shows our estimate of the stellar (chromospheric) $\mathrm{H} \alpha$ emission produced by the WTTS PTFO 8-8695 in the continuumnormalized spectrum. The dashed line shows a spectrum of AD Leo (dM3e) rotationally broadened to the same $v \sin i$ as PTFO 8-8695. The smooth red profile shows the spectrum of AD Leo in which the $\mathrm{H} \alpha$ emission has been multiplied by a factor of 2.4 after first rotationally broadening the spectrum to the same $v \sin i$ as PTFO 8-8695.

position is changing the most rapidly. This may indicate that there is some smearing of the profile from the relatively long exposure times compared to the expected orbital period.

\subsection{Kitt Peak Data}

The first two nights (2012 December 8-9) of the Kitt Peak observations of PTFO 8-8695 are shown in Figures 5 and 6. In the figures, the individual profiles are shown in the black histograms, and the average nightly profile is overplotted in red on each of the individual profiles. In contrast to the McDonald observations shown in Figure 2, there is no clear excess emission observed at the predicted velocity position of the planetary companion to the star. Some weak variation in the $\mathrm{H} \alpha$ line is observed on both nights; however, the velocity positions of these changes appear well within the range expected for the stellar chromospheric emission. These variations are likely caused by spatially localized chromospheric emission features on the surface of this magetically active WTTS, and are probably unrelated to the potential planetary companion. The $\mathrm{H} \alpha$ emission on these two nights, presumably stellar in origin, is somewhat weaker than seen in the McDonald data: the emission equivalent widths are $8.33 \pm 0.03 \AA$ and $7.93 \pm 0.06 \AA$ on 2012 December 8 and 9 respectively. Since no excess $\mathrm{H} \alpha$ emission is clearly detected on these nights, we do not attempt to measure any quantities related to this and so no data is reported for the excess equivalent width, velocity centroid, or line width in Table 2.

On the third night of the Kitt Peak run, the behavior of the $\mathrm{H} \alpha$ line in PTFO 8-8695 became much more active, showing similar behavior to that seen in the McDonald data. The $\mathrm{H} \alpha$ profiles from this night (2012 December 10) are shown in Figure 7, where starting with the fourth exposure (UT 5:53) obvious excess emission was detected in the $\mathrm{H} \alpha$ line that again appears to move with the velocity of the planetary companion predicted by van Eyken et al. (2012). We estimate the stellar chromospheric emission using the profile obtained at UT 7:34 on this night when the planet is predicted to be most redshifted. To minimize the effects of potential stellar $\mathrm{H} \alpha$ fluctuations

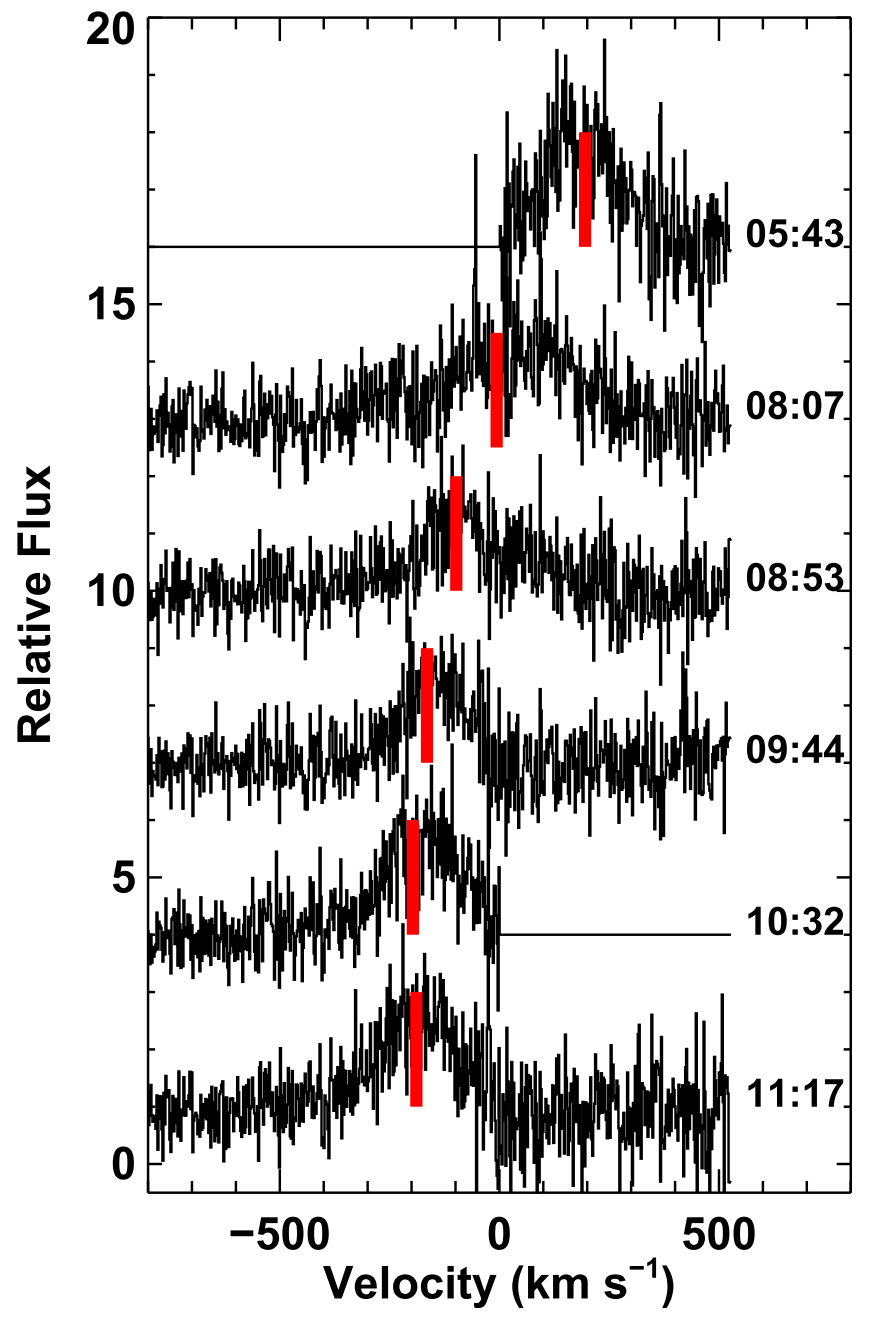

Figure 4. Plots of the six observed continuum-normalized $\mathrm{H} \alpha$ profiles of PTFO 8-8695 obtained on 2013 November 15 UT with the stellar (chromospheric) component subtracted out, again with the UT time of the midpoint of each observation given. The thick, red vertical line on each profile again marks the expected velocity position of the planetary companion detected by van Eyken et al. (2012) from transit observations of this WTTS.

such as those seen in Figures 5 and 6, we use this profile to estimate the stellar component of the line since it is close in time to the profiles showing the excess emission. The UT 7:34 profile is reflected about zero velocity to create an estimate of the stellar chromospheric profile. This estimated chromospheric profile has an equivalent width of $8.57 \pm 0.12 \AA$.

In an effort to isolate the non-stellar excess emission, we subtract the estimated chromospheric from all the spectra observed on night 3 , and the resulting profiles are shown in Figure 8 . We then performed the same measurements of excess emission equivalent width, velocity centroid, and line width as described above for the McDonald data. These values are given in Table 2. For the first 2 observations on this night, we do not report the excess emission velocity centroid or line width. Both of these quantities are computed by effectively dividing by the excess equivalent width. Because the excess emission is very weak and not securely detected in these first two observations, the values and uncertainties in the velocity centroid and line width become extremely large and provide no constraints on any of the analysis. 


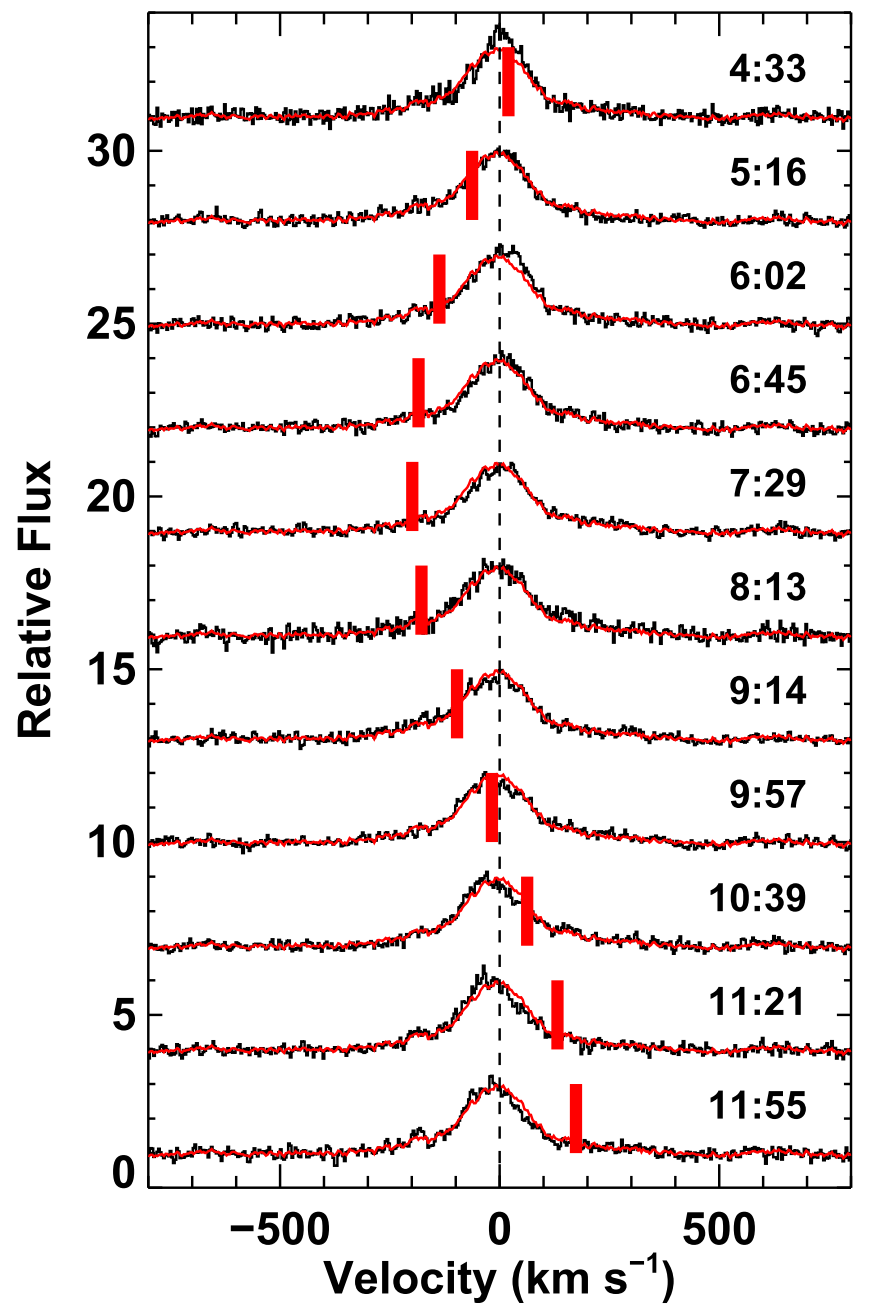

Figure 5. The 11 observed continuum-normalized H $\alpha$ profiles of PTFO 8-8695 obtained on 2012 December 8 UT at Kitt Peak Observatory are shown in the black histograms with the UT time (midpoint) of each observation. The smooth red curve shows the average $\mathrm{H} \alpha$ profile from this night. The thick, red vertical line on each profile marks the expected velocity position of the planetary companion detected by van Eyken et al. (2012) from transit observations of this WTTS. No clear excess emission is seen in the line profile on this night.

\subsection{Revisiting the HET and Keck Data}

As mentioned in Section 3.1, the HET and Keck data collected in 2011 do not show any obvious relation to the predicted velocity position of the planet. This can be made clearer by attempting a stellar subtraction similar to that done to produce Figures 4 and 8 . For these figures, we were able to use profiles from the same night to represent the stellar component, but in the case of the HET and Keck data there is only one observation per night and there is substantial variation from one observation to the next. Since the profiles on the first two Kitt Peak nights show no apparent excess emission, we take the average of these 21 spectra to represent the stellar emission. This stellar component appears to vary in strength between the McDonald and Kitt Peak observations, and examinations of the profiles in Figure 1 suggest it does here as well. Therefore, we scaled this stellar component so that it matched the observed profiles in Figure 1 as best it could, but such that the stellar profile was never above the observed profile. The implicit assumption is that the observed profile is composed of a stellar (including a chromosphere) component plus potentially an

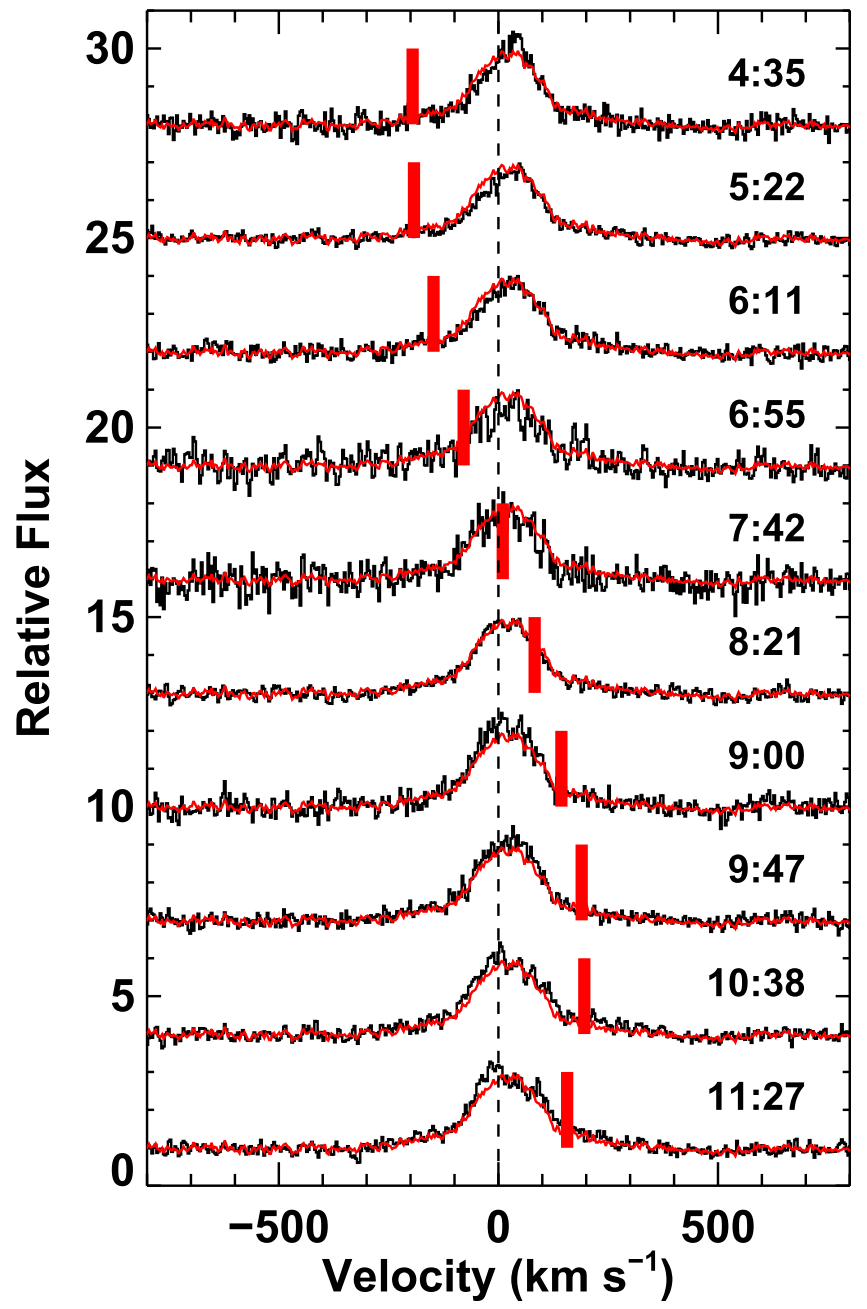

Figure 6. The 10 observed continuum-normalized H $\alpha$ profiles of PTFO 8-8695 obtained on 2012 December 9 UT at Kitt Peak Observatory are shown in the black histograms with the UT (midpoint) time of each observation. The smooth red curve shows the average $\mathrm{H} \alpha$ profile from this night. The thick, red vertical line on each profile marks the expected velocity position of the planetary companion detected by van Eyken et al. (2012) from transit observations of this WTTS. No clear excess emission is seen in the line profile on this night.

excess emission component on top of this. Once we subtract the scaled stellar profiles, only the excess emission is observed and these profiles are shown in Figure 9. While some profiles appear to subtract nearly to zero (e.g., phase 127.792), there is substantial excess emission in many of them. As mentioned above, in some cases the excess emission component is close in velocity position to the predicted velocity of the planet, while in other cases it is far away and shows no obvious connection to the suspected planet. However, given that substantial time elapsed between each of the spectra shown in Figure 9 (and 1), we cannot be certain how these profiles evolved. Below, in Section 4.3.4, we offer an interpretation that attempts to account for all the observed profile shapes from this star.

\subsection{Orbital Fits}

The observations of the RV variations of the excess $\mathrm{H} \alpha$ emission recorded at McDonald Observatory and at Kitt Peak, densely sampled in time, lend themselves to an exploration of possible orbital motion. Figure 10 shows the measured velocity centroids of the excess $\mathrm{H} \alpha$ emission against orbital phase based 


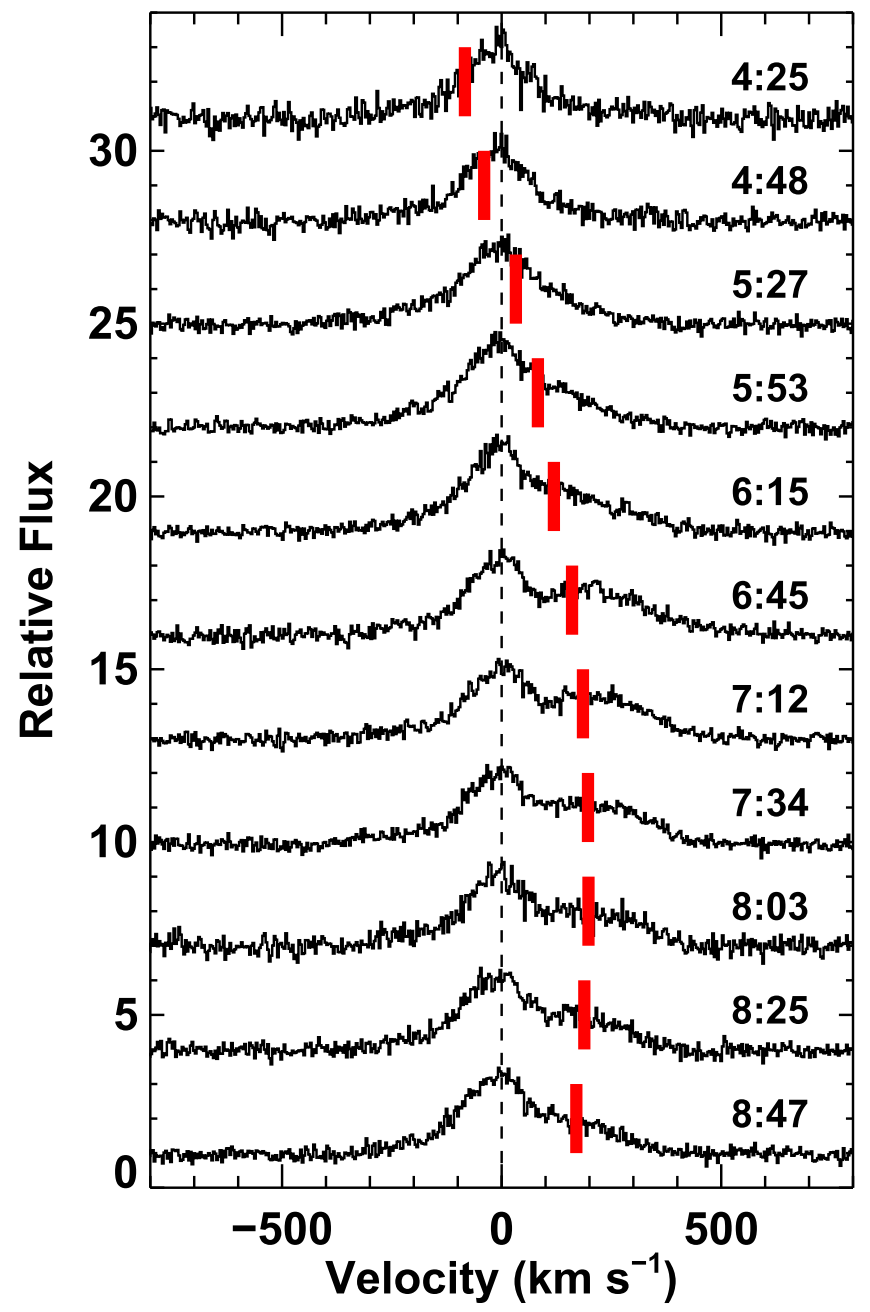

Figure 7. 11 observed continuum-normalized $\mathrm{H} \alpha$ profiles of PTFO 8-8695 obtained on 2012 December 10 UT at Kitt Peak Observatory are plotted with the UT time (midpoint) of each observation. The thick, red vertical line on each profile marks the expected velocity position of the planetary companion detected by van Eyken et al. (2012) from transit observations of this WTTS. Starting at UT 5:53 there is clear excess $\mathrm{H} \alpha$ emission coincident in velocity space at the radial velocity of the planet.

on the ephemeris in van Eyken et al. (2012). While Barnes et al. (2013) revise the ephemeris of PTFO 8-8695, they do not provide a unique solution; the results depend on the assumed stellar mass. However, the ephemerides of Barnes et al. result in a maximum phase offset of 0.026 for the data presented here, which is likely negligible compared to other uncertainties in the measurements and analysis discussed below. Therefore, we use the ephemeris of van Eyken et al. (2012) throughout and plot (red line) the predicted velocity curve of the planet in Figure 10 using their orbital parameters, which assume a circular orbit. The value of $\chi^{2}$ for this velocity curve is 607 , and the reduce $\chi^{2}$ value is $\chi_{r}^{2}=41$. The measured velocity variations generally track the expected orbital motion of the planet, with the McDonald observations somewhat closer to the predicted curve than those from Kitt Peak. We discuss below the possibility that the excess $\mathrm{H} \alpha$ emission includes components not purely in orbit with the planet which might lead to this difference; however, for completeness, we proceed here assuming these velocity variations are the result of orbital motion.

The RV observations presented in Figure 10 were obtained in 2012 and 2013, while the ephemeris in van Eyken et al.

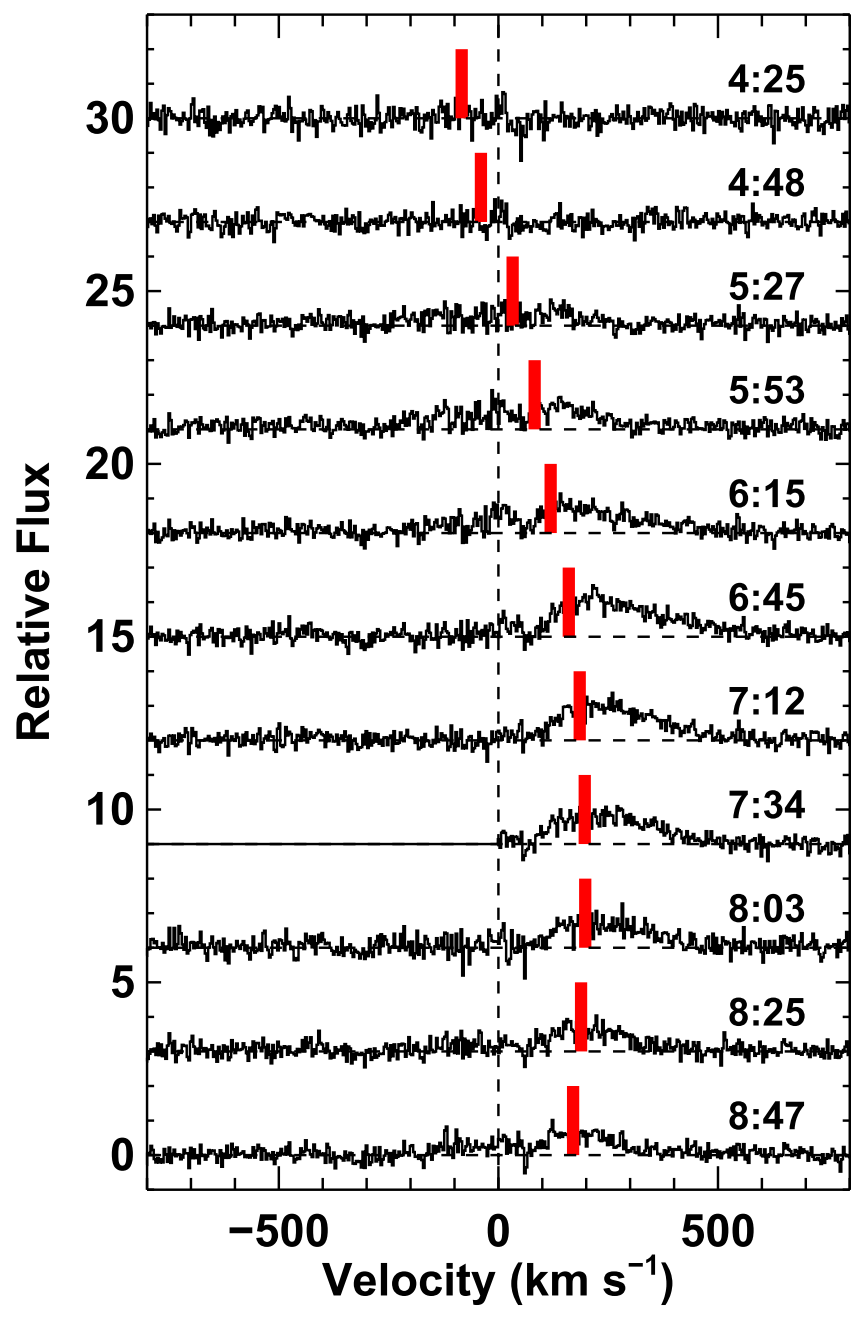

Figure 8. 11 observed continuum-normalized H $\alpha$ profiles of PTFO 8-8695 obtained on 2012 December 10 UT with the stellar (chromospheric) component subracted out are plotted. The thick, red vertical line on each profile marks the expected velocity position of the planetary companion detected by van Eyken et al. (2012) from transit observations of this WTTS. Starting at UT 5:53 there is clear excess $\mathrm{H} \alpha$ emission coincident in velocity space at the radial velocity of the planet. Some weak emission also appears to be present at UT 5:27.

(2012) is based on transit data taken in 2009 and 2010, so there is some uncertainty in the predicted phasing of the RV observations presented here. Ciardi et al. (2015) analyze additional transit data for PTFO 8-8695 including photometry from the Spitzer satellite. The transits recovered in Ciardi et al. (2015) were offset from the predicted times using the discovery ephemeris, but were all within the original ephemeris uncertainty from van Eyken et al. (2012). We can use the offset to the observed Spitzer transit (data obtained in 2012 April) as an estimate of the uncertainty in the ephemeris when comparing the predicted phases of the RV observations in Figure 10. The green curve in this plot shows the predicted RV curve of the planet using the orbit determined in van Eyken et al. (2012), shifted to match the transit midpoint observed by Spitzer in 2012 (Ciardi et al. 2015). The shift is only 0.026 in phase. This emphemeris fits the $\mathrm{H} \alpha \mathrm{RV}$ variations more poorly, giving $\chi^{2}=889$ and $\chi_{r}^{2}=60$. This may indicate a problem with the Spitzer transit time determination or, as discussed in Section 4, may be be due to the $\mathrm{H} \alpha$ emitting gas not strictly moving with the candidate planet around this star. 


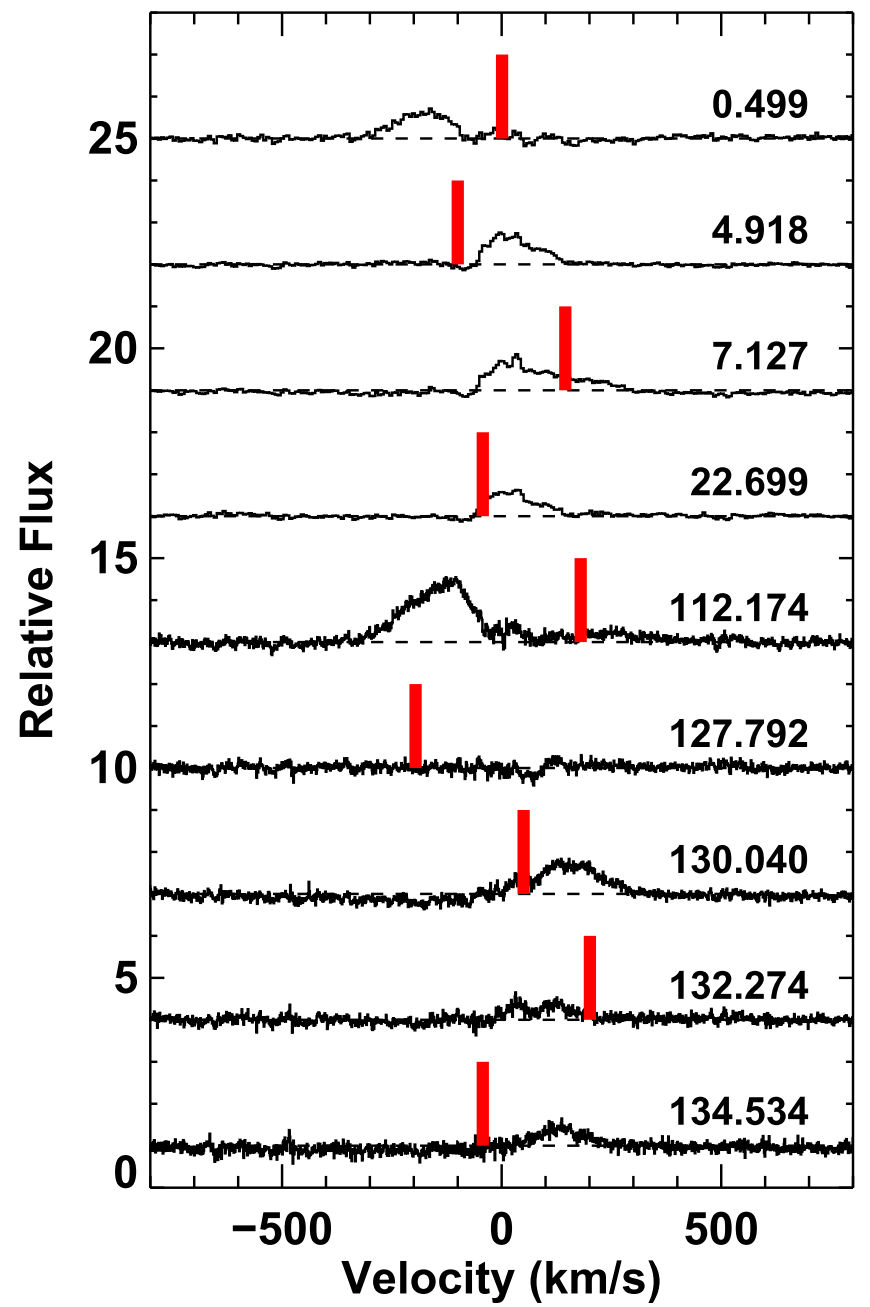

Figure 9. Continuum-normalized $\mathrm{H} \alpha$ profiles of PTFO 8-8695 obtained with the HET (top four profiles) and with Keck (bottom five profiles) from Figure 1 after an estimate of the stellar component has been subtracted off. The thick, red vertical line on each profile marks the expected velocity position of the planetary companion detected by van Eyken et al. (2012) from transit observations of this WTTS.

Assuming that the excess $\mathrm{H} \alpha \mathrm{RV}$ variations shown in Figure 10 result from the orbital motion of the planet around PTFO 8-8695, we fit a sine wave (circular orbit) to the RV points (solid black line in the figure; $\chi^{2}=570$ and $\chi_{r}^{2}=41$ ), obtaining a velocity semi-amplitude of $211.9 \pm 22.1 \mathrm{~km} \mathrm{~s}^{-1}$. Clearly, the pure sine wave fit and predicted sine curves (green and red lines) of Figure 10 are not good fits to all the observed $\mathrm{RV}$ variations of the excess $\mathrm{H} \alpha$ emission, although they do a fairly good job of fitting the data from McDonald Observatory in 2013. We can obtain a better fit using a full Keplerian curve, allowing the orbit to be eccentric. This fit is shown in the blue line of Figure $10\left(\chi^{2}=177\right.$ and $\left.\chi_{r}^{2}=16\right)$. In addition to fitting the RV points, we used the transit time as an additionl constraint. To do so, we assume the transit midpoint of van Eyken et al. (2012) as the observed transit time and assign an uncertainty equal to the phase offset between this original transit time and that determined from the Spitzer observations of Ciardi et al. (2015). The resulting velocity semi-amplitude is $K \sin i=268.1 \pm 13.4 \mathrm{~km} \mathrm{~s}^{-1}$, with an eccentricity of $e=0.35 \pm 0.12$. While such a large eccentricity brings the putative planet closer to the star, the planet is still outside the

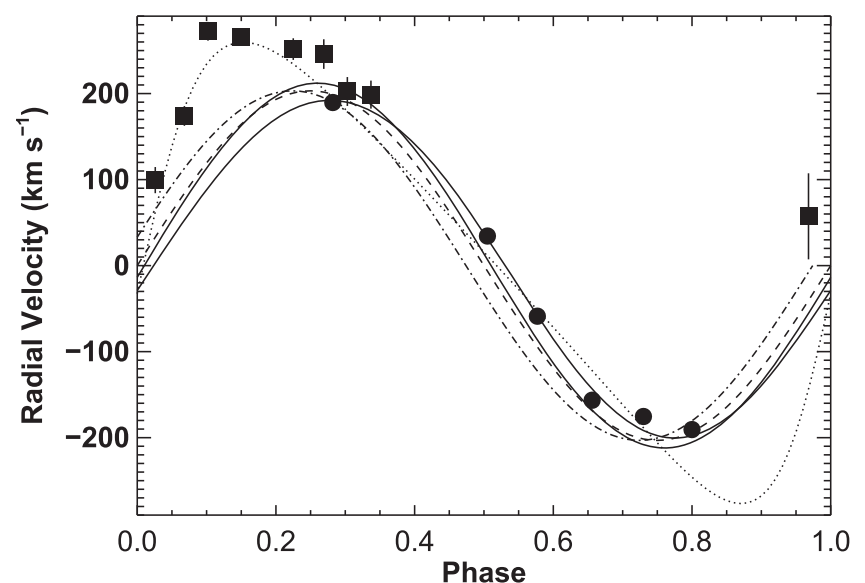

Figure 10. Solid symbols show the measured RV of the excess $\mathrm{H} \alpha$ emission from PTFO 8-8695, including uncertainties, as a function of orbital phase using the ephemeris from van Eyken et al. (2012). Circles show data from McDonald Observatory and squares show data from Kitt Peak National Observatory. The dashed line shows the predicted planetary velocity curve using the ephemeris from van Eyken et al. (2012). The dashed-dotted line shows the predicted RV curve from van Eyken et al. (2012) shifted in phase to account for the revised transit center epoch determined from Spitzer data by Ciardi et al. (2015). The solid line is a circular orbit fit to all the measured RV points. The dotted line is a full Keplerian fit to all the measured RV points, including the transit midpoint time as a constraint (see text). Finally, the dashed-triple dot line is a full Keplerian fit to only the McDonald RV points, still using the transit midpoint time as a constraint.

star at periastron for the largest stellar radius $\left(1.07 R_{\odot}\right)$ found by either van Eyken et al. (2012) or Barnes et al. (2013), although nominally the surface of the planet would pass within one planetary radius of the stellar surface. We suggest below that there may be a significant non-orbital contribution to the excess $\mathrm{H} \alpha \mathrm{RV}$ variations, so a full orbital analysis is likely not warranted. As a result we do not report other parameters of the orbital fit. We note that using Equation (18) of Gu et al. (2003) that the planet's orbit should be circularized in $10^{3}-10^{5}$ years depending on the exact value of the planetary quality factor $Q_{p}^{\prime}$, so we do not expect an eccentric orbit for the suspected planet, unless that eccentricity is being excited by a third body in the system. Below we argue that the excess $\mathrm{H} \alpha$ emission observed in the Kitt Peak spectra may be particularly affected by nonorbital motion, so we perform a full Keplerian fit to the McDonald RVs only, again using the transit time as a constraint. This fit is shown in the purple line in Figure 10 and gives $K \sin i=196.2 \pm 5.6 \mathrm{~km} \mathrm{~s}^{-1}$ with an eccentricity of $e=0.02 \pm 0.05$. The total $\chi^{2}=14$, which is substantially improved, whereas $\chi_{r}^{2}=7.0$ which is also an improvement, but only modestly so due to the small number of degrees of freedom given only six RV data points to which the orbit is fit.

\section{DISCUSSION}

We have detected variable $\mathrm{H} \alpha$ emission from the young transiting planet candidate PTFO 8-8695. This object sometimes shows a component of $\mathrm{H} \alpha$ emission that appears to be in excess to the stellar chromospheric emission. At times, the excess $\mathrm{H} \alpha$ appears at a random phase relative to the expected velocity position of the claimed planetary companion (Figures 1 and 9). However, at other times the excess $\mathrm{H} \alpha$ emission appears to move in wavelength as would be expected if it were produced by the suspected planetary companion. This raises the intriguing possibility that the excess $\mathrm{H} \alpha$ emission is associated 
with the planetary companion. In general though, there are at least three potential sources of this variable $\mathrm{H} \alpha$ emission that should be considered. The emission could be associated with (1) the star itself, (2) accretion flows from a tenuous disk, or (3) it could be directly related to a low-mass companion.

\subsection{A Stellar Source: Magnetic Activity and Flaring}

PTFO 8-8695 is a young $\mathrm{T}$ Tauri star, and as such is expected to be very magnetically active. Such stars produce variable chromospheric $\mathrm{H} \alpha$ emission (e.g., Hatzes 1995). If all of the observed $\mathrm{H} \alpha$ emission is taken to be from the star, then the measured emission equivalent width when it is active would be $\sim 18 \AA$. This would give PTFO 8-8695 a log $\left(L_{\mathrm{H} \alpha} / L_{\mathrm{bol}}\right)=-2.92$ which would make it stronger than every other magnetically active $\mathrm{M}$ star in the sample of Hawley et al. (1996). Additionally, the rotationally broadened and enhanced chromospheric profile of the dMe flare star AD Leo matches well the core of the $\mathrm{H} \alpha$ line from PTFO 8-8695 (Figure 3). While there is clearly some variability in this core as seen in Figures 5 and 6, the strong, highly Doppler-shifted excess $\mathrm{H} \alpha$ emission seen in Figures 2 and 7 cannot be explained by chromospheric emission on the surface of the star.

However, a stellar flare could potentially produce such Doppler-shifted emission, at least in principle. Stellar flares in dMe stars often produce significant, nearly symmetric line broadening at the base of $\mathrm{H} \alpha$. The resulting line profile routinely shows the standard narrow chromospheric emission on top of a very broad (FWHM of a few hundred $\mathrm{km} \mathrm{s}^{-1}$ ) base of emission (e.g., Eason et al. 1992; Jones et al. 1996) reminisent of some of the weaker line profiles shown in Figure 1 (e.g., at phases 4.918, 7.127, 22.699, and 127.174). It is very rare in a flare to see dramatically asymmetric emission with a highly red- or blueshifted component nearly equal in strength to the central chromospheric emission as seen in the profiles of Figures 2 and 7.

T Tauri stars in general are known to flare (e.g., Gahm 1990; Guenther \& Ball 1999). A potentially better analog of the type of variable $\mathrm{H} \alpha$ emission expected from chromospheric emission and flaring on PTFO 8-8695 is the WTTS V410 Tau, with $v \sin i=77.7 \mathrm{~km} \mathrm{~s}^{-1}$ (e.g., Carroll et al. 2012) compared to the measured $v \sin i=80.6 \pm 8.1 \mathrm{~km} \mathrm{~s}^{-1}$ for PTFO 8-8695 (van Eyken et al. 2012). The $\mathrm{H} \alpha$ emission equivalent width ( $<3 \AA$ with a typical value $\sim 1-2 \AA$ ) on V410 Tau (e.g., Hatzes 1995; Fernández et al. 2004; Mekkaden et al. 2005) is weaker than seen on PTFO 8-8695, although V410 Tau has an earlier spectral type that raises the continuum level without necessarily affecting the strength of the chromospheric emission. V410 Tau has been observed to flare in a number of studies. Outside of flares, the $\mathrm{H} \alpha$ line of V410 Tau is fairly symmetric, relatively narrow, and is similar in shape to the chromospheric $\mathrm{H} \alpha$ profiles for PTFO 8-8695 seen in Figures 3, 5, and 6 (Hatzes 1995; Fernández et al. 2004; Skelly et al. 2010). The $\mathrm{H} \alpha$ line of V410 Tau can grow much stronger and broader during a flare, and also show asymmetries; however, the observed asymmetries seen during flares do not show excess emission with apparent peaks shifted out to greater than $\pm 200 \mathrm{~km} \mathrm{~s}^{-1}$ (Hatzes 1995; Rice et al. 2011) as seen here in PTFO 8-8695. The typical pattern in a flare is for the line to very rapidly (timescale of a few minutes) strengthen and broaden with only a slight asymmetry developing. The strength and width of the line then decay exponentially with a timescale of $\sim 1 \mathrm{hr}$ for strong flares (e.g., Fernández et al. 2004). This is not the temporal behavior observed in PTF08-8695. There is at least one additional piece of evidence against the flaring interpretation for the excess $\mathrm{H} \alpha$ emission seen in PTFO 8-8695. Whenever V410 Tau shows flare emission in $\mathrm{H} \alpha$, significant He I $5876 \AA$ emission also appears. This He I line is covered in the echelle formats of both our McDonald and Kitt Peak data. We have searched both data sets for evidence of this emission, including co-adding the spectra when the $\mathrm{H} \alpha$ emission appears stationary (UT 9:44 to 11:17 for McDonald; UT 6:45 to 8:25 for Kitt Peak) to increase the signal-to noise. No evidence of He I emission is seen. Lastly, if the observed excess $\mathrm{H} \alpha$ emission seen in Figures 2 and 7 were the result of a stellar flare, it would be a remarkable coincidence that the flareinduced asymmetry just happened to appear at and move with the same velocity position in the line profile as that expected for the planetary companion. In particular, the motion shown in Figures 2 and 4 where the excess emission first appears strongly on one side of the line profile and then moves to the other side has not to our knowledge been observed in the $\mathrm{H} \alpha$ emission of flare stars. Flares have been observed on PTFO 8-8695 (van Eyken et al. 2012; Ciardi et al. 2015), and while flares on this star likely will produce changes in the strength and shape of the $\mathrm{H} \alpha$ emission line, we believe all the points described above argue strongly against a purely stellar origin.

\subsection{A Disk Accretion Source}

Another possibility is that the $\mathrm{H} \alpha$ emission arises from material accreting onto the star from a tenuous disk that may still surround PTFO 8-8695. While this star is classified as a WTTS, it is at the boundary between WTTSs and accreting CTTSs (Briceño et al. 2005), although no dust is evident in its infrared spectral energy distribution, including Spitzer data out to $24 \mu \mathrm{m}$ (Hernández et al. 2007). If we take the average excess $\mathrm{H} \alpha$ equivalent width and estimate an accretion rate using the empirical calibrations in Fang et al. (2009), we find a value of $\sim 3 \times 10^{-10} M_{\odot} \mathrm{yr}^{-1}$, which is relatively low compared to the full sample in Fang et al. If there is weak accretion from a tenuous disk, it is probable that such a disk would be detected in infrared emission as even very tenuous disks that feed very low accretion rates on the order of $10^{-11} M_{\odot} \mathrm{yr}^{-1}$ produce a detectable near-IR excess (Gillen et al. 2014). This accretion rate is significantly less than the value estimated above, suggesting that if the $\mathrm{H} \alpha$ emission resulted from disk accretion, PTFO 8-8695 should show an IR excess unless there has been substantial grain growth around this 2-3 Myr old star which has removed essentially all the small grains.

If PTFO 8-8695 is accreting material onto the star from a gas disk devoid of small grains, the excess $\mathrm{H} \alpha$ emission may result entirely from the accreting material whether or not there is a planetary companion present. This accretion-related emission would presumably be similar to $\mathrm{H} \alpha$ emission seen in other CTTSs, many of which also have close companions. If there is a low-mass companion to PTFO 8-8695, accretion from a disk may be through accretion streams such as those proposed by Artymowicz \& Lubow (1996) (see also Günther \& Kley 2002). At this time, it is not known if a planetary mass companion can excite accretion streams such as those modeled by Artymowicz \& Lubow (1996) and Günther \& Kley (2002). A few CTTSs binaries are thought to potentially be accreting through accretion streams. These include DQ Tau (Basri et al. 1997; Mathieu et al. 1997), UZ Tau E (Jensen et al. 2007), AK Sco (Alencar et al. 2003), KH 15D (Hamilton et al. 2012), and the 
eclipsing binary system CoRoT 223992193 in NGC 2264 (Gillen et al. 2014). None of these stars shows the type of $\mathrm{H} \alpha$ variations seen in PTFO 8-8695 where the accretion-related emission appears to move from one side of the line profile to the other as it spirals onto one or both of the stars. This type of line profile behavior is also not seen in the $\mathrm{H} \alpha$ profile variations of single CTTSs in extensive studies of their line profile variability (e.g., Giampapa et al. 1993; Johns \& Basri 1995a, 1995b; Johns-Krull \& Basri 1997; Oliveira et al. 1998; Alencar et al. 2001), nor is it predicted from theoretical models of magnetospheric accretion such as those shown in Kurosawa \& Romanova (2013). While we cannot completely rule out accretion from a tenuous disk as the source of the excess $\mathrm{H} \alpha$ emission observed in PTFO 8-8695, we argue that this is not the most likely explanation of the observed emission. Deep mid-IR or millimeter continuum observations, or a deep search for close circumstellar disk gas emission (e.g., $\mathrm{H}_{2}$ emission, see France et al. 2012), could shed light on whether there is a tenuous disk around this star feeding accretion onto it.

\subsection{A Planetary Companion Source}

Particularly given the RV variations of the excess emission component of the $\mathrm{H} \alpha$ line, the most likely explanation is that this emission arises from an orbiting companion.

\subsubsection{Mass Estimates}

Assuming the companion hypothesis, we can use these measured RV variations with the orbital fits performed above to estimate the mass of the system. van Eyken et al. (2012) did not positively detect RV motion of PTFO 8-8695 itself; however, if we adopt their upper limit on the reflex motion of the star $\left(2.13 \pm 0.12 \mathrm{~km} \mathrm{~s}^{-1}\right)$, we can use Kepler's laws to find an upper limit for the total mass in the system of $\left(M_{*}+M_{P}\right) \sin ^{3} i=0.456 \pm 0.082 \quad M_{\odot}$ assuming a circular orbit (black curve in Figure 10). Alternatively, if we use the full Keplerian solution and again apply the upper limit on the stellar reflex motion of the star from van Eyken et al. (assuming that this is the upper limit for the velocity semi-amplitude, $K$ ), we obtain an upper mass limit for the system of $\left(M_{*}+M_{P}\right) \sin ^{3} i=0.539 \pm 0.047 M_{\odot}$. Finally, if we restrict ourselves to the fit to the McDonald-only data, we find an upper limit to the mass of $\left(M_{*}+M_{P}\right) \sin ^{3} i=0.362 \pm 0.018$ $M_{\odot}$. We can then use these total mass estimates with the observed ratio of the companion and stellar RV amplitudes, again adopting the upper limit on the stellar RV amplitude of the star from van Eyken et al., to estimate the mass of the companion. Using the parameters from the circular orbit fit gives $M_{P} \sin ^{3} i=4.75 \pm 0.56 M_{\mathrm{JUP}}$, while using the parameters from the full Keplerian fit gives $M_{P} \sin ^{3} i=4.45 \pm 0.34 M_{\mathrm{JUP}}$, and the McDonald-only fit giving $M_{P} \sin ^{3} i=4.07 \pm 0.45$ $M_{\mathrm{JUP}}$, all clearly in the planetary range. van Eyken et al. (2012) determine an orbital inclination of $61.8 \pm 3.7$ which then gives a total mass for the system of $0.666 \pm 0.108 M_{\odot}$ for the circular orbit, $0.787 \pm 0.095 M_{\odot}$ for the eccentric orbit, and $0.529 \pm 0.027 M_{\odot}$ for the McDonald-only orbital fit. The planet mass then becomes $6.94 \pm 0.92 M_{\mathrm{JUP}}$ for the circular fit, $6.50 \pm 0.76 M_{\mathrm{JUP}}$ for the eccentric fit, and $5.95 \pm 0.66 M_{\mathrm{JUP}}$ for the McDonald-only fit. Again, this is an upper limit to the planet's mass given that the RV variations measured for the star are only upper limits on the reflex motion of the orbit. Indeed,
Ciardi et al. (2015) present new K band based RV measurements of the star, estimating a new stellar RV semi-amplitude of $K=0.370 \pm 0.333 \mathrm{~km} \mathrm{~s}^{-1}$, which would lower the estimate of the planet's true mass to $\sim 1.1 M_{\mathrm{JUP}}$. The true stellar RV variations could be even smaller which would imply a still lower planet mass, although if the planet's mass is too low its Roche radius would become smaller than the planet and it should then be losing substantial mass.

The estimates given above for the actual mass of the planet depend on the inclination of the planet's orbit, which is uncertain. van Eyken et al. (2012) determine an orbital inclination of $61.8 \pm 3.7$; however, Barnes et al. (2013) argue that this inclination changes over time as a result of nodal precession of the orbit. They find the value of the orbital inclination can change from $\sim 25^{\circ}$ to $90^{\circ}$ with a period in the range of $300-500$ days. Thus, there is the possibility the planet's orbital inclination at the time of the observations presented here was significantly different from the 61.8 assumed. Assuming $i=61^{\circ} .8 \pm 3.7$, the stellar mass inferred from the circular orbit fit is $M_{*}=0.659 \pm 0.108 M_{\odot}$. The effective temperature of PTFO 8-8695 is $3470 \mathrm{~K}$ (Briceño et al. 2005). Using the pre-main sequence evolutionary tracks of Siess et al. (2000), this corresponds to a mass of $\sim 0.35 M_{\odot}$ at an age of $2 \mathrm{Myr}$ (the tracks of Baraffe et al. 2015 give a mass of $\sim 0.32 M_{\odot}$ at the same temperature and age). Thus, our measured mass is larger than the predicted masses by $\sim 3 \sigma$. It is well established that theoretical pre-main sequence tracks underestimate the true mass of young stars at these masses (Hillenbrand \& White 2004). According to these authors, premain sequence tracks routinely predict a mass that is $30 \%$ lower than the true mass. Thus, if the true mass is $0.659 M_{\odot}$, we might expect evolutionary tracks to give a mass of 0.461 $M_{\odot}$. With an uncertainty of $0.108 M_{\odot}$, the actual predicted masses from the evolutionary tracks are $1.0 \sigma-1.3 \sigma$ lower than this value. Thus, while our inferred mass is higher than the predicted mass, the difference is within the expectations given the known systematic trends in the evolutionary tracks at these low masses. No matter what the true inclination is, our measured mass will be larger than the mass inferred from evolutionary tracks. For example, if the inclination is $90^{\circ}$, the measured mass would be $0.451 M_{\odot}$. While this is larger than the mass inferred from the tracks, it is larger by $\sim 30 \%$, which is the typical difference found for young stars in this mass range. If the orbital inclination at the time of the spectroscopic observations presented here was as low as $40^{\circ}$, this would imply true stellar mass of $1.7 M_{\odot}$, which corresponds to an effective temperature of $\sim 4670 \mathrm{~K}$, or a spectral type between K3 and K4, using the Siess et al. (2000) evolutionary tracks at 2 Myr. This is very inconsistent with the observed spectral type. Thus, we infer that the orbital inclination at the time of the spectroscopic observations could not be much less than $61^{\circ} .8$.

\subsubsection{Production of Excess $H \alpha$ Emission}

If the excess $\mathrm{H} \alpha$ emission does come from the planetary companion, how is it produced? One of the most remarkable aspects of the apparent excess $\mathrm{H} \alpha$ emission is its strength. Tables 1 and 2 show that, when visible, the planet's $\mathrm{H} \alpha$ equivalent width is typically $70 \%-80 \%$ that from the star. Because the excess equivalent width and the stellar chromospheric equivalent width are measured relative to the same stellar continuum, the $\mathrm{H} \alpha$ luminosity from the planet then reaches $80 \%$ that from the star. The $10.5 \AA$ emission equivalent 
width from the star, while stronger than the dM3e star AD Leo as shown in Figure 3, is not atypical of very magnetically active $\mathrm{dM} 3-4 \mathrm{e}$ stars, several of which have $\mathrm{H} \alpha$ emission equivalent widths of $10.0 \AA$ or more (e.g., Hawley et al. 1996). However, the planet orbiting PTFO 8-8695 is much smaller than the star - the apparent area of the planet is $\sim 3.4 \%$ that of the star (van Eyken et al. 2012). In order to produce an $\mathrm{H} \alpha$ equivalent width that is $\sim 75 \%$ that of the star, the $\mathrm{H} \alpha$ surface flux of the planet would have to be 22 times that of the star. We do not know the effective temperature or spectral type of the young planet orbiting PTFO 8-8695; however, it is likely cooler and later in type than the star. Such a dramatic rise in $\mathrm{H} \alpha$ surface flux is not observed in active stars or brown dwarfs with spectral types later than M4 (e.g., Schmidt et al. 2010; West et al. 2011). As a result, it is doubtful the emission from the planet around PTFO 8-8695 is produced by magnetic activity.

We further argue that the emission from this planet does not arise on its surface at all, but instead either in a confined region around the planet such as in the planetary magnetosphere, or in a more complicated flow with the planet feeding the material. Similar gas flows have been observed in absorption (e.g., Cauley et al. 2015; Ehrenreich et al. 2015) and have been modeled by a number of investigators (e.g., Matsakos et al. 2015). The radius of the planet is in the range of 1.64 $R_{\text {JUP }}$ (Barnes et al. 2013) to $1.91 R_{\text {JUP }}$ (van Eyken et al. 2012). Assuming the planet's rotation is tidally locked with its orbit (the synchronization timescale is estimated to be $\ll 1 \mathrm{Myr}$ using relationships given in $\mathrm{Gu}$ et al. 2003), the maximum $v \sin i$ the planet could have is $18.6-21.7 \mathrm{~km} \mathrm{~s}^{-1}$. However, the average velocity width of the excess $\mathrm{H} \alpha$ emission is $87.3 \pm 4.9 \mathrm{~km} \mathrm{~s}^{-1}$, significantly more than can be accounted for by the rotation of the planet. Instead, we suggest the most likely explanation is that the emission results from mass outflow from the planet.

\subsubsection{Mass Loss from the Companion}

Mass outflow from a hot Jupiter was first detected by VidalMadjar et al. (2003) and these observations have been confirmed by several additional studies (e.g., Linsky et al. 2010). Mass loss from hot Jupiters has been studied theoretically by a number of investigators (e.g., Lammer et al. 2003; Baraffe et al. 2004, 2006; Murray-Clay et al. 2009; Lai et al. 2010; Adams 2011; Trammell et al. 2011). van Eyken et al. (2012) noted that this planet's radius is very close to its Roche lobe radius. Using Equation (1) of van Eyken et al. (2012) and the best-fitting parameters of Barnes et al. (2013) actually places the Roche radius of the planet slightly inside the inferred radius of the planet. Thus, the potential planet orbiting PTFO 8-8695 is a prime candidate for significant mass loss. Using the best-fit parameters from Barnes et al. (2013), the escape velocity from the planet is $82-88 \mathrm{~km} \mathrm{~s}^{-1}$, which is very comparable to the velocity width of the excess $\mathrm{H} \alpha$ emission apparently associated with the planet, further suggestive that this planet may be losing considerable mass.

Murray-Clay et al. (2009) was the first to theoretically study mass loss from a hot Jupiter orbiting a young pre-main sequence star. These authors note that the very high mass loss rates from $\mathrm{T}$ Tauri stars will likely completely stifle planetary flows on the day side of the hot Jupiter as the result of the large ram pressure associated with the stellar wind. However, the large mass loss rates used by Murray-Clay et al. (2009) are only really appropriate for CTTSs which are still accreting material from a disk. Stellar wind mass loss rates appropriate for a WTTS such as PTFO 8-8695 have not yet been determined. The semimajor axis of the suspected companion to PTFO 8-8695 is less than $\sim 2 R_{\odot}$ while the star itself has a radius of $\sim 1 R_{\odot}$ (van Eyken et al. 2012; Barnes et al. 2013). Thus, the planet would be orbiting only about 1 stellar radius above the stellar surface. $\mathrm{T}$ Tauri stars are measured to have average surface magnetic fields of $2-3 \mathrm{kG}$ (Johns-Krull 2007), so it is likely that the planet is orbiting inside the Alfvén radius of the star, in which case Murray-Clay et al. (2009) suggest the outflow from both the day and night sides of the planet may be suppressed. However, the planetary magnetosphere may help balance the stellar magnetic pressure in order to allow the wind from the planet to get started, and it may also lead to the outflowing material building up substantial optical depth at a size that is several times the nominal planetary radius (e.g., Trammell et al. 2011), which is needed in order to produce the amount of $\mathrm{H} \alpha$ emission seen as discussed above. Once the material escapes the planet and its magnetosphere, it may then be the stellar field that ultimately controls where the material flows.

Most models of mass loss from a hot Jupiter find that the flow ultimately gets redirected by the stellar wind with the material eventually leaving the system. Here though, the planetary wind would be launched inside the region of space governed by the stellar magnetosphere. In the case of CTTSs, much of the material flowing off the disk near the co-rotation radius is forced by the stellar magnetosphere to travel along the field lines and accrete onto the star (e.g., Bouvier et al. 2007). van Eyken et al. (2012) find that the stellar rotation period is locked to the candidate planet's orbital period, so the planet would be feeding material into the stellar magnetosphere at the co-rotation radius, which is very analogous to CTTSs accretion models (e.g., Shu et al. 1994). It may then be that material flowing off the planet is accreting onto the star in a magnetospheric accretion flow similar to that in CTTSs. This accreting material might then produce emission at higher redshifted velocities as it accelerates in the gravitational potential well of the star, as modeled for example by Matsakos et al. (2015). Such higher velocity tails are hinted at in Figures 7 and 8. Material that is lost into a wind may also contribute $\mathrm{H} \alpha$ emission at velocities different from the orbital velocity of the suspected planet.

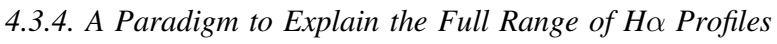

The interplay of material being lost by the planet embedded in the magnetosphere of a young star might explain all the $\mathrm{H} \alpha$ variations observed in PTFO 8-8695. Line profiles seen in Figures 1, 5, and 6 suggest that there are times when only emission from the chromospherically active star is present. As mentioned above, PTFO 8-8695 is known to flare. A strong stellar flare may rapidly strip away the outflowing material from the planet resulting in no excess emission for some time. Due to flares or other dynamo-related variability, the planet at times may be in regions dominated by open stellar field lines where a strong stellar wind is flowing which stifles the planetary outflow as suggested by Murray-Clay et al. (2009). At such times, there may be no excess $\mathrm{H} \alpha$ emission associated with the planet. At some point after a flare or once the planet moves into a region of closed stellar fields, the planetary outflow may be re-established and we observe the excess emission associated with the planet. As the flow continues and 
material begins to fill the stellar magnetosphere, accrete onto the star, or be lost to a wind, $\mathrm{H} \alpha$ emission will begin to have contributions from gas no longer directly tied to the planet. The RV structure of this gas as viewed from Earth, and the resulting line profile, might then get quite complex and produce the various profile shapes observed in Figures 1 and 9, as well as producing the higher velocity tails seen in Figures 5 and 6 . The possibility that some component of the $\mathrm{H} \alpha$ excess emission is not directly tied to the planet cautions that the eccentric Keplerian orbit discussed above may not be real, but simply due to the difficulty in isolating the emission coming directly from the planet. Indeed, since the RV variations traced by the excess $\mathrm{H} \alpha$ emission likely include components not strictly in orbit with the suspected planet, it is difficult to definitively rule out any of the specific orbital fits shown in Figure 10.

When the stellar field adjusts itself due to another flare or some dynamo variation, the sequence outlined above could then start all over again. Barnes et al. (2013) suggest that the planet's orbital plane is inclined relative to the stellar equatorial plane, so even though the stellar rotation and planet's orbit may be locked, the planet is still moving in latitude relative to the star and hence is moving through different parts of the stellar magnetosphere. This motion through the stellar magnetic field could produce changes in a planetary flow within even a single orbit, leading to complicated variations in the $\mathrm{H} \alpha$ line profile. To explain the full range of variation in the line profiles observed from PTFO 8-8695, we are forced to invoke accretion onto the star. As discussed above, there is no suggestion of an infrared excess in this star, even out to $24 \mu \mathrm{m}$. Feeding the accretion flow with material escaping from a planet naturally explains the lack of an infrared excess, and the profile variability in PTFO 8-8695 is at times well matched assuming excess $\mathrm{H} \alpha$ emission that is physically associated with the reported planet around this star. Thus, we find the planet scenario outlined above the most likely explanation for the variations we see in the lines. Above, we crudely estimated that the excess $\mathrm{H} \alpha$ emission would imply an accretion rate of $3 \times 10^{-10} M_{\odot} \mathrm{yr}^{-1}$ onto the star. If this accretion rate is fed entirely by a planet of $\sim 7 M_{\mathrm{JUP}}$ as suggested above, the lifetime of the planet would be $\sim 2 \times 10^{7}$ years. Thus, if the candidate planet orbiting PTFO 8-8695 is real, we expect it to evolve substantially over the pre-main sequence lifetime of this star.

\section{SUMMARY}

We have used relatively high time cadence, high spectral resolution optical observations to detect excess $\mathrm{H} \alpha$ emission from the 2-3 Myr old WTTS PTFO 8-8695. At some times these high cadence observations show that the excess emission appears to move in velocity as expected if it were produced by the suspected planetary companion to this young star. We have considered the possibility that the observed excess emission is produced by stellar activity (flares), accretion from a disk, or from a planetary companion; we find the planetary companion to be the most likely explanation. Yu et al. (2015) recently examined additional photometry and spectroscopy of this star in an effort to test the planet hypothesis for this system. They do not favor the hot Jupiter hypothesis, instead suggesting that their data point to either starspots, eclipses by circumstellar dust (fed by either a circumstellar disk or a low-mass evaporating planet), or occultations of an accretion hotspot as the most likely explanation for the variations they observe. However, these authors did not observe the strong, variable, excess Balmer emission that we discuss here and their alternatives would not account for it. Above, we discuss the difficulties associated with a stellar activity or disk accretion origin for the $\mathrm{H} \alpha$ variations, and we conclude that an evaporating planet is the best explanation for the variations we observe. While no single model may fit all the data on this star, this may be due to the extreme nature of this object as a very rapidly rotating, magnetically active pre-main sequence star. Therefore, we believe the planetary companion hypothesis is still a viable component of this unique system.

If the excess $\mathrm{H} \alpha$ emission we see does come from a planetary companion, the strength of the emission indicates that it arises in an extended volume around the planet, likely fed by mass loss from the planet which is expected to be overflowing its Roche lobe. Interpreting the RV variations of the excess $\mathrm{H} \alpha$ emission as coming from the planet, we place an upper limit on the mass of the star as $M_{*} \sin ^{3} i=0.535 \pm 0.047 M_{\odot}$, while the planet's mass would then be $M_{P} \sin ^{3} i=4.45 \pm 0.34 M_{\text {JUP. }}$. While there is evidence that the orbital inclination of this system varies dramatically due to nodal precession (Barnes et al. 2013; Ciardi et al. 2015), the inclination at the time of our spectroscopic observations cannot be too low or the stellar mass would become very inconsistent with its spectral type. This leads to an upper limit for the planet's mass of $\sim 7 M_{\text {JUP. }}$. While the observations presented here are highly suggestive that we have directly observed the spectroscopic signature of a mass losing planet in orbit around this young star, these results are primarily based on relatively low signal-to-noise observations. Further high signal-to-noise, high cadence time resolved $\mathrm{H} \alpha$ observations over several predicted orbits will greatly aid in solidifying the picture outlined in this work. In addition, we caution that theoretical work is likely still needed to determine whether such strong emission could really be produced by a young hot Jupiter undergoing mass loss.

L.P. and C.M.J.-K. wish to acknowledge partial support for this research from the NASA Origins of Solar Systems program through grant number 07-SSO07-86 made to Lowell Observatory. CMJ-K also wishes to acknowledge partial support for this work from the National Science Foundation through grant number 1212122 made to Rice University. This work was funded in part through the 2013-2014 NAU/NASA Space Grant Undergraduate Research Internship for Jacob $\mathrm{N}$. McLane. Finally, we wish to thank an anonymous referee for several useful comments that improved the original manuscript.

\section{REFERENCES}

Adams, F. C. 2011, ApJ, 730, 27

Aigrain, S., Hodgkin, S., Irwin, J., et al. 2007, MNRAS, 375, 29

Alencar, S. H. P., Johns-Krull, C. M., \& Basri, G. 2001, AJ, 122, 3335

Alencar, S. H. P., Melo, C. H. F., Dullemond, C. P., et al. 2003, A\&A, 409, 1037

Artymowicz, P., \& Lubow, S. H. 1996, ApJL, 467, L77

Baraffe, I., Alibert, Y., Chabrier, G., \& Benz, W. 2006, A\&A, 450, 1221

Baraffe, I., Homeier, D., Allard, F., \& Chabrier, G. 2015, A\&A, 577, A42

Baraffe, I., Selsis, F., Chabrier, G., et al. 2004, A\&A, 419, L13

Barnes, J. W., van Eyken, J. C., Jackson, B. K., Ciardi, D. R., \& Fortney, J. J. 2013, ApJ, 774, 53

Basri, G., Johns-Krull, C. M., \& Mathieu, R. D. 1997, AJ, 114, 781

Bertout, C. 1989, ARA\&A, 27, 351

Bodenheimer, P., Hubickyj, O., \& Lissauer, J. J. 2000, Icar, 143, 2

Boss, A. P. 1997, Sci, 276, 1836

Boss, A. P. 1998, ApJ, 503, 923 
Bouvier, J., Alencar, S. H. P., Harries, T. J., Johns-Krull, C. M., \& Romanova, M. M. 2007, in Protostars and Planets V, ed. B. Reipurth, D. Jewitt, \& K. Keil (Tucson, AZ: Univ. Arizona Press), 479

Bowler, B. P., Liu, M. C., Shkolnik, E. L., \& Dupuy, T. J. 2013, ApJ, 774, 55

Briceño, C., Calvet, N., Hernández, J., et al. 2005, AJ, 129, 907

Cameron, A. G. W. 1978, M\&P, 18, 5

Carroll, T. A., Strassmeier, K. G., Rice, J. B., \& Künstler, A. 2012, A\&A, 548, AA95

Cauley, P. W., Redfield, S., Jensen, A. G., et al. 2015, arXiv:1507.05916

Charbonneau, D., Brown, T. M., Burrows, A., \& Laughlin, G. 2007, in Protostars and Planets V, ed. B. Reipurth, D. Jewitt, \& K. Keil (Tucson, AZ: Univ. Arizona Press), 701

Ciardi, D. R., van Eyken, J. C., Barnes, J. W., et al. 2015, ApJ, 809, 42

Cochran, W. D., Hatzes, A. P., \& Paulson, D. B. 2002, AJ, 124, 565

Cody, A. M., Stauffer, J., Baglin, A., et al. 2014, AJ, 147, 82

Cody, A. M., Tayar, J., Hillenbrand, L. A., Matthews, J. M., \& Kallinger, T. 2013, AJ, 145, 79

Crockett, C. J., Mahmud, N. I., Prato, L., et al. 2011, ApJ, 735, 78

Crockett, C. J., Mahmud, N. I., Prato, L., et al. 2012, ApJ, 761, 164

Delorme, P., Gagné, J., Girard, J. H., et al. 2013, A\&A, 553, L5

Dodson-Robinson, S. E., Veras, D., Ford, E. B., \& Beichman, C. A. 2009, ApJ, 707, 79

Durisen, R. H., Boss, A. P., Mayer, L., et al. 2007, in Protostars and Planets V, ed. B. Reipurth, D. Jewitt, \& K. Keil (Tucson, AZ: Univ. Arizona Press), 607

Eason, E. L. E., Giampapa, M. S., Radick, R. R., Worden, S. P., \& Hege, E. K. 1992, AJ, 104, 1161

Ehrenreich, D., Bourrier, V., Wheatley, P. J., et al. 2015, Natur, 522, 459

Esposito, M., Guenther, E., Hatzes, A. P., \& Hartmann, M. 2006, in Coll. Tenth Anniversary of 51 Peg-b: Status of and Prospects for Hot Jupiter Studies, ed. L. Arnold et al. (Paris: Frontier Group)

Fang, M., van Boekel, R., Wang, W., et al. 2009, A\&A, 504, 461

Fernández, M., Stelzer, B., Henden, A., et al. 2004, A\&A, 427, 263

Fischer, D. A., \& Valenti, J. 2005, ApJ, 622, 1102

France, K., Schindhelm, E., Herczeg, G. J., et al. 2012, ApJ, 756, 171

Gahm, G. F. 1990, in IAU Symp. 137, Flare Stars in Star Clusters, Associations and the Solar Vicinity, ed. L. V. Mirzoyan, B. R. Petterson, \& M. K. Tsvetkov (Dordrecht: Kluwer), 193

Giampapa, M. S., Basri, G. S., Johns, C. M., \& Imhoff, C. 1993, ApJS, 89, 321

Gillen, E., Aigrain, S., McQuillan, A., et al. 2014, A\&A, 562, A50

Gonzalez, G. 1997, MNRAS, 285, 403

Gu, P.-G., Lin, D. N. C., \& Bodenheimer, P. H. 2003, ApJ, 588, 509

Guenther, E. W., \& Ball, M. 1999, A\&A, 347, 508

Günther, R., \& Kley, W. 2002, A\&A, 387, 550

Hamilton, C. M., Johns-Krull, C. M., Mundt, R., Herbst, W., \& Winn, J. N. 2012, ApJ, 751, 147

Hatzes, A. P. 1995, ApJ, 451, 784

Hawley, S. L., Gizis, J. E., \& Reid, I. N. 1996, AJ, 112, 2799

Hernández, J., Calvet, N., Briceño, C., et al. 2007, ApJ, 671, 1784

Hillenbrand, L. A., \& White, R. J. 2004, ApJ, 604, 741

Hinkle, K., Wallace, L., Valenti, J., \& Harmer, D. 2000, in Visible and Near Infrared Atlas of the Arcturus Spectrum 3727-9300 A, ed. Kenneth. Hinkle, Lloyd. Wallace, \& Jeff. Valenti, (San Francisco: ASP), 2000

Hubickyj, O., Bodenheimer, P., \& Lissauer, J. J. 2005, Icar, 179, 415

Huélamo, N., Figueira, P., Bonfils, X., et al. 2008, A\&A, 489, L9

Huerta, M., Johns-Krull, C. M., Prato, L., Hartigan, P., \& Jaffe, D. T. 2007 ApJ, 678, 472

Ireland, M. J., Kraus, A., Martinache, F., Law, N., \& Hillenbrand, L. A. 2011, ApJ, 726, 113

Jensen, E. L. N., Dhital, S., Stassun, K. G., et al. 2007, AJ, 134, 241

Johns, C. M., \& Basri, G. 1995a, AJ, 109, 2800

Johns, C. M., \& Basri, G. 1995b, ApJ, 449, 341

Johns-Krull, C. M. 2007, ApJ, 664, 975

Johns-Krull, C. M., \& Basri, G. 1997, ApJ, 474, 433
Jones, B. F., Fischer, D. A., \& Stauffer, J. R. 1996, AJ, 112, 1562

Kraus, A. L., \& Ireland, M. J. 2012, ApJ, 745, 5

Kraus, A. L., Ireland, M. J., Cieza, L. A., et al. 2014, ApJ, 781, 20

Kuiper, G. P. 1951, in Symp. Proc. 50th Anniversary of the Yerkes Observatory and Half a Century of Progress in Astrophysics, ed. J. A. Hynek (New York, NY: McGraw-Hill), 357

Kurosawa, R., \& Romanova, M. M. 2013, MNRAS, 431, 2673

Lafrenière, D., Jayawardhana, R., \& van Kerkwijk, M. H. 2008, ApJL, 689, L153

Lai, D., Helling, C., \& van den Heuvel, E. P. J. 2010, ApJ, 721, 923

Lammer, H., Selsis, F., Ribas, I., et al. 2003, ApJL, 598, L121

Levison, H. F., Morbidelli, A., Gomes, R., \& Backman, D. 2007, in Protostars and Planets V, ed. B. Reipurth, D. Jewitt, \& K. Keil (Tucson, AZ: Univ. Arizona Press), 669

Linsky, J. L., Yang, H., France, K., et al. 2010, ApJ, 717, 1291

Lissauer, J. J., \& Stevenson, D. J. 2007, in Protostars and Planets V, ed B. Reipurth, D. Jewitt, \& K. Keil (Tucson, AZ: Univ. Arizona Press), 591 Luhman, K. L., Wilson, J. C., Brandner, W., et al. 2006, ApJ, 649, 894

Mahmud, N. I., Crockett, C. J., Johns-Krull, C. M., et al. 2011, ApJ, 736, 123

Marois, C., Macintosh, B., Barman, T., et al. 2008, Sci, 322, 1348

Mathieu, R. D., Stassun, K., Basri, G., et al. 1997, AJ, 113, 1841

Matsakos, T., Uribe, A., \& Königl, A. 2015, A\&A, 578, A6

Mayor, M., \& Queloz, D. 1995, Natur, 378, 355

Mekkaden, M. V., Pukalenthi, S., Muneer, S., \& Bastian, A. B. 2005, BASI 33, 433

Miller, A. A., Irwin, J., Aigrain, S., Hodgkin, S., \& Hebb, L. 2008, MNRAS, 387,349

Murray-Clay, R. A., Chiang, E. I., \& Murray, N. 2009, ApJ, 693, 23

Neuhäuser, R., Errmann, R., Berndt, A., et al. 2011, AN, 332, 547

Neuhäuser, R., Guenther, E. W., Wuchterl, G., et al. 2005, A\&A, 435, L13

Neuhäuser, R., Mugrauer, M., Seifahrt, A., et al. 2008, A\&A, 484, 281

Nguyen, D. C., Brandeker, A., van Kerkwijk, M. H., \& Jayawardhana, R. 2012, ApJ, 745, 119

Oliveira, J. M., Unruh, Y. C., Foing, B. H. \& MUSICOS 96 Collaboration 1998, Ap\&SS, 261, 143

Papaloizou, J. C. B., Nelson, R. P., Kley, W., Masset, F. S., \& Artymowicz, P. 2007, in Protostars and Planets V, ed. B. Reipurth, D. Jewitt, \& K. Keil (Tucson, AZ: Univ. Arizona Press), 655

Paulson, D. B., \& Yelda, S. 2006, PASP, 118, 706

Pollack, J. B., Hubickyj, O., Bodenheimer, P., et al. 1996, Icar, 124, 62

Prato, L., Huerta, M., Johns-Krull, C. M., et al. 2008, ApJL, 687, L103

Ramsey, L. W., Adams, M. T., Barnes, T. G., et al. 1998, Proc. SPIE, 3352, 34

Rice, J. B., Strassmeier, K. G., \& Kopf, M. 2011, ApJ, 728, 69

Saar, S. H., \& Donahue, R. A. 1997, ApJ, 485, 319

Santos, N. C., Israelian, G., \& Mayor, M. 2004, A\&A, 415, 1153

Schmidt, S. J., West, A. A., Hawley, S. L., \& Pineda, J. S. 2010, AJ, 139, 1808

Schmidt, T. O. B., Neuhäuser, R., Seifahrt, A., et al. 2008, A\&A, 491, 311

Setiawan, J., Henning, T., Launhardt, R., et al. 2008, Natur, 451, 38

Setiawan, J., Weise, P., Henning, Th., et al. 2007, ApJL, 660, L145

Shu, F., Najita, J., Ostriker, E., et al. 1994, ApJ, 429, 781

Siess, L., Dufour, E., \& Forestini, M. 2000, A\&A, 358, 593

Skelly, M. B., Donati, J.-F., Bouvier, J., et al. 2010, MNRAS, 403, 159

Trammell, G. B., Arras, P., \& Li, Z.-Y. 2011, ApJ, 728, 152

Tull, R. G. 1998, Proc. SPIE, 3355, 387

Tull, R. G., MacQueen, P. J., Sneden, C., \& Lambert, D. L. 1995, PASP, 107,251

Valenti, J. A. 1994, PhD thesis, Univ. California Berkeley

van Eyken, J. C., Ciardi, D. R., Rebull, L. M., et al. 2011, AJ, 142, 60

van Eyken, J. C., Ciardi, D. R., von Braun, K., et al. 2012, ApJ, 755, 42

Vidal-Madjar, A., Lecavelier des Etangs, A., Désert, J.-M., et al. 2003, Natur, 422, 143

Vogt, S. S., Allen, S. L., Bigelow, B. C., et al. 1994, Proc. SPIE, 2198, 362

West, A. A., Morgan, D. P., Bochanski, J. J., et al. 2011, AJ, 141, 97

Yu, L., Winn, J. N., Gillon, M., et al. 2015, ApJ, 812, 48 